\title{
A GENERALIZATION OF THE SUBSPACE THEOREM WITH POLYNOMIALS OF HIGHER DEGREE
}

\author{
JAN-HENDRIK EVERTSE AND ROBERTO G. FERRETTI
}

To Professor Wolfgang Schmidt on his 70th birthday

\begin{abstract}
Recently, Corvaja and Zannier [2, Theorem 3] proved an extension of the Subspace Theorem with polynomials of arbitrary degree instead of linear forms. Their result states that the set of solutions in $\mathbb{P}^{n}(K)$ ( $K$ number field) of the inequality being considered is not Zariski dense.

In this paper we prove, by a different method, a generalization of their result, in which the solutions are taken from an arbitrary projective variety $X$ instead of $\mathbb{P}^{n}$. Further we give a quantitative version, which states in a precise form that the solutions with large height lie in a finite number of proper subvarieties of $X$, with explicit upper bounds for the number and for the degrees of these subvarieties (Theorem 1.3 below).

We deduce our generalization from a general result on twisted heights on projective varieties (Theorem 2.1] in Section 21). Our main tools are the quantitative version of the Absolute Parametric Subspace Theorem by Evertse and Schlickewei [5. Theorem 1.2], as well as a lower bound by Evertse and Ferretti [4, Theorem 4.1] for the normalized Chow weight of a projective variety in terms of its $m$-th normalized Hilbert weight.
\end{abstract}

\section{INTRODUCTION}

1.1. The Subspace Theorem can be stated as follows. Let $K$ be a number field (assumed to be contained in some given algebraic closure $\overline{\mathbb{Q}}$ of $\mathbb{Q}$ ), $n$ a positive integer, $0<\delta \leqslant 1$ and $S$ a finite set of places of $K$. For $v \in S$, let $L_{0}^{(v)}, \ldots, L_{n}^{(v)}$ be linearly independent linear forms in $\overline{\mathbb{Q}}\left[x_{0}, \ldots, x_{n}\right]$. Then

2000 Mathematics Subject Classification: 11J68, 11J25.

Keywords and Phrases: Diophantine approximation, Subspace Theorem. 
the set of solutions $\mathbf{x} \in \mathbb{P}^{n}(K)$ of

$$
\log \left(\prod_{v \in S} \prod_{i=0}^{n} \frac{\left|L_{i}^{(v)}(\mathbf{x})\right|_{v}}{\|\mathbf{x}\|_{v}}\right) \leqslant-(n+1+\delta) h(\mathbf{x})
$$

is contained in the union of finitely many proper linear subspaces of $\mathbb{P}^{n}$.

Here, $h(\cdot)$ denotes the absolute logarithmic height on $\mathbb{P}^{n}(\overline{\mathbb{Q}}),|\cdot|_{v},\|\cdot\|_{v}$ $(v \in S)$ denote normalized absolute values on $K$ and normalized norms on $K^{n+1}$, and each $|\cdot|_{v}$ has been extended to $\overline{\mathbb{Q}}$ (see $\$ 1.4$ below). The Subspace Theorem was first proved by Schmidt [14, [15] for the case that $S$ consists of the archimedean places of $K$, and then later extended by Schlickewei [13] to the general case.

1.2. We state a generalization of the Subspace Theorem in which the linear forms $L_{i}^{(v)}$ are replaced by homogeneous polynomials of arbitrary degree, and in which the solutions are taken from an $n$-dimensional projective subvariety of $\mathbb{P}^{N}$ where $N \geqslant n \geqslant 1$.

By a projective subvariety of $\mathbb{P}^{N}$ we mean a geometrically irreducible Zariski-closed subset of $\mathbb{P}^{N}$. For a Zariski-closed subset $X$ of $\mathbb{P}^{N}$ and for a field $\Omega$, we denote by $X(\Omega)$ the set of $\Omega$-rational points of $X$. For homogeneous polynomials $f_{1}, \ldots, f_{r}$ in the variables $x_{0}, \ldots, x_{N}$ we denote by $\left\{f_{1}=0, \ldots, f_{r}=0\right\}$ the Zariski-closed subset of $\mathbb{P}^{N}$ given by $f_{1}=0, \ldots$, $f_{r}=0$.

Then our result reads as follows:

Theorem 1.1. Let $K$ be a number field, $S$ a finite set of places of $K$ and $X$ a projective subvariety of $\mathbb{P}^{N}$ defined over $K$ of dimension $n \geqslant 1$ and degree d. Let $0<\delta \leqslant 1$. Further, for $v \in S$ let $f_{0}^{(v)}, \ldots, f_{n}^{(v)}$ be a system of homogeneous polynomials in $\overline{\mathbb{Q}}\left[x_{0}, \ldots, x_{N}\right]$ such that

$$
X(\overline{\mathbb{Q}}) \cap\left\{f_{0}^{(v)}=0, \ldots, f_{n}^{(v)}=0\right\}=\emptyset \text { for } v \in S .
$$

Then the set of solutions $\mathbf{x} \in X(K)$ of the inequality

$$
\log \left(\prod_{v \in S} \prod_{i=0}^{n} \frac{\left|f_{i}^{(v)}(\mathbf{x})\right|_{v}^{1 / \operatorname{deg} f_{i}^{(v)}}}{\|\mathbf{x}\|_{v}}\right) \leqslant-(n+1+\delta) h(\mathbf{x})
$$


is contained in a finite union $\bigcup_{i=1}^{u}\left(X \cap\left\{G_{i}=0\right\}\right)$, where $G_{1}, \ldots, G_{u}$ are homogeneous polynomials in $K\left[x_{0}, \ldots, x_{N}\right]$ not vanishing identically on $X$ of degree at most

$$
(8 n+6)(n+2)^{2} d \Delta^{n+1} \delta^{-1} \text { with } \Delta:=\operatorname{lcm}\left(\operatorname{deg} f_{i}^{(v)}: v \in S, 0 \leqslant i \leqslant n\right) .
$$

It should be noted that if $N=n, X=\mathbb{P}^{n}$ and $f_{0}^{(v)}, \ldots, f_{n}^{(v)}$ are linear forms, then condition (1.2) means precisely that $f_{0}^{(v)}, \ldots, f_{n}^{(v)}$ are linearly independent.

We give an immediate consequence:

Corollary 1.2. Let $f_{0}, \ldots, f_{n}$ be homogeneous polynomials in $\overline{\mathbb{Q}}\left[x_{0}, \ldots, x_{n}\right]$ such that

$$
\left\{\mathbf{x} \in \overline{\mathbb{Q}}^{n+1}: f_{0}(\mathbf{x})=\cdots=f_{n}(\mathbf{x})=0\right\}=\{\mathbf{0}\} .
$$

Let $0<\delta \leqslant 1$. Then the set of solutions $\mathbf{x}=\left(x_{0}, \ldots, x_{n}\right) \in \mathbb{Z}^{n+1}$ of

$$
\prod_{i=0}^{n}\left|f_{i}(\mathbf{x})\right|^{1 / \operatorname{deg} f_{i}} \leqslant\left(\max _{0 \leqslant i \leqslant n}\left|x_{i}\right|\right)^{-\delta}
$$

is contained in some finite union of hypersurfaces $\left\{G_{1}=0\right\} \cup \cdots \cup\left\{G_{u}=0\right\}$, where each $G_{i}$ is a homogeneous polynomial in $\mathbb{Q}\left[x_{0}, \ldots, x_{n}\right]$ of degree at most $(8 n+6)(n+2)^{2} \Delta^{n+1} \delta^{-1}$ with $\Delta:=\operatorname{lcm}\left(\operatorname{deg} f_{i}: 0 \leqslant i \leqslant n\right)$.

1.3. In their paper [6], Faltings and Wüstholz introduced a new method to prove the Subspace Theorem, and gave some examples showing that their method enables to prove extensions of the Subspace Theorem with higher degree polynomials instead of linear forms, and with solutions from an arbitrary projective variety. Ferretti [7, [8] observed the role of Mumford's degree of contact [10] (or the Chow weight, see $\$ 2.3$ below) in the work of Faltings and Wüstholz and worked out several other cases. Evertse and Ferretti [4] showed that the extensions of the Subspace Theorem as proposed by Faltings and Wüstholz can be deduced directly from the Subspace Theorem itself.

Recently, Corvaja and Zannier [2, Theorem 3] obtained a result similar to our Theorem 1.1] with $X=\mathbb{P}^{n}$. (More precisely, Corvaja and Zannier gave an essentially equivalent affine formulation, in which the polynomials 
$f_{i}^{(v)}$ need not be homogeneous and in which the solutions x have $S$-integer coordinates). In fact, Corvaja and Zannier showed that the set of solutions of (1.3) is contained in a finite union of hypersurfaces in $\mathbb{P}^{n}$ and gave some further information about the structure of these hypersurfaces, on the other hand they did not provide an explicit bound for their degrees. Corvaja and Zannier stated their result only for the case $X=\mathbb{P}^{n}$ but with their methods this may be extended to the case that $X$ is a complete intersection. In contrast, our result is valid for arbitrary projective subvarieties $X$ of $\mathbb{P}^{N}$.

In their paper [2], Corvaja and Zannier proved also finiteness results for several classes of Diophantine equations. It is likely, that similar results can be deduced by means of our approach, but we have not gone into this.

1.4. Below we state a quantitative version of Theorem 1.1. We first introduce the necessary notation. All number fields considered in this paper are contained in a given algebraic closure $\overline{\mathbb{Q}}$ of $\mathbb{Q}$. Let $K$ be a number field and denote by $G_{K}$ the Galois group of $\overline{\mathbb{Q}}$ over $K$. For $\mathbf{x}=\left(x_{0}, \ldots, x_{N}\right) \in \overline{\mathbb{Q}}^{N+1}$, $\sigma \in G_{K}$ we write $\sigma(\mathbf{x})=\left(\sigma\left(x_{0}\right), \ldots, \sigma\left(x_{N}\right)\right)$. Denote by $M_{K}$ the set of places of $K$. For $v \in M_{K}$, choose an absolute value $|.|_{v}$ normalized such that the restriction of $|\cdot|_{v}$ to $\mathbb{Q}$ is $|\cdot|^{\left[K_{v}: \mathbb{R}\right] /[K: \mathbb{Q}]}$ if $v$ is archimedean and $|\cdot|_{p}^{\left[K_{v}: \mathbb{Q}_{p}\right] /[K: \mathbb{Q}]}$ if $v$ lies above the prime number $p$. Here $|$.$| is the ordinary absolute value,$ and $|\cdot|_{p}$ is the $p$-adic absolute value with $|p|_{p}=p^{-1}$. These absolute values satisfy the product formula $\prod_{v \in M_{K}}|x|_{v}=1$ for $x \in K^{*}$.

Given $\mathbf{x}=\left(x_{0}, \ldots, x_{N}\right) \in K^{N+1}$ we put $\|\mathbf{x}\|_{v}:=\max \left(\left|x_{0}\right|_{v}, \ldots,\left|x_{N}\right|_{v}\right)$ for $v \in M_{K}$. Then the absolute logarithmic height of $\mathbf{x}$ is defined by $h(\mathbf{x})=$ $\log \left(\prod_{v \in M_{K}}\|\mathbf{x}\|_{v}\right)$. By the product formula, $h(\lambda \mathbf{x})=h(\mathbf{x})$ for $\lambda \in K^{*}$. Moreover, $h(\mathbf{x})$ depends only on $\mathbf{x}$ and not on the choice of the particular number field $K$ containing $x_{0}, \ldots, x_{N}$. Thus, this function $h$ gives rise to a height on $\mathbb{P}^{N}(\overline{\mathbb{Q}})$.

Given a system $f_{0}, \ldots, f_{m}$ of polynomials with coefficients in $\overline{\mathbb{Q}}$ we define $h\left(f_{0}, \ldots, f_{m}\right):=h(\mathbf{a})$, where $\mathbf{a}$ is a vector consisting of the non-zero coefficients of $f_{0}, \ldots, f_{m}$. Further by $K\left(f_{0}, \ldots, f_{m}\right)$ we denote the extension of $K$ generated by the coefficients of $f_{0}, \ldots, f_{m}$. The height of a projective subvariety $X$ of $\mathbb{P}^{N}$ defined over $\overline{\mathbb{Q}}$ is defined by $h(X):=h\left(F_{X}\right)$, where $F_{X}$ is the Chow form of $X$ (see $\S 2.3$ below). 
For every $v \in M_{K}$ we choose an extension of $|\cdot|_{v}$ to $\overline{\mathbb{Q}}$ (this amounts to extending $|\cdot|_{v}$ to the algebraic closure $\bar{K}_{v}$ of $K_{v}$ and choosing an embedding of $\overline{\mathbb{Q}}$ into $\left.\bar{K}_{v}\right)$. Further for $v \in M_{K}, \mathbf{x}=\left(x_{0}, \ldots, x_{N}\right) \in \overline{\mathbb{Q}}^{N+1}$ we put $\|\mathbf{x}\|_{v}:=\max \left(\left|x_{0}\right|_{v}, \ldots,\left|x_{N}\right|_{v}\right)$.

1.5. Schmidt [16] was the first to obtain a quantitative version of the Subspace Theorem, giving an explicit upper bound for the number of subspaces containing all solutions with 'large' height. Since then his basic result has been improved and generalized in various directions. Evertse and Schlickewei [5, Theorem 3.1] deduced a quantitative version of the Absolute Subspace Theorem, dealing with solutions in $\mathbb{P}^{n}(\overline{\mathbb{Q}})$ of some absolute extension of (1.1). Their result can be stated as follows.

Let again $K$ be a number field, and $S$ a finite set of places of $K$ of cardinality $s$. Let $n \geqslant 1,0<\delta \leqslant 1$. For $v \in S$, let $L_{0}^{(v)}, \ldots, L_{n}^{(v)}$ be linearly independent linear forms in $\overline{\mathbb{Q}}\left[x_{0}, \ldots, x_{n}\right]$. Put $\mathcal{D}:=\prod_{v \in S}\left|\operatorname{det}\left(L_{0}^{(v)}, \ldots, L_{n}^{(v)}\right)\right|_{v}$ and assume that $\left[K\left(L_{i}^{(v)}\right): K\right] \leqslant C$ for $v \in S, i=0, \ldots, n$. Then the set of $\mathbf{x} \in \mathbb{P}^{n}(\overline{\mathbb{Q}})$ with

$$
\begin{gathered}
\log \left(\mathcal{D}^{-1} \prod_{v \in S} \prod_{i=0}^{n} \max _{\sigma \in G_{K}} \frac{\left|L_{i}^{(v)}(\sigma(\mathbf{x}))\right|_{v}}{\|\sigma(\mathbf{x})\|_{v}}\right) \leqslant-(n+1+\delta) h(\mathbf{x}), \\
h(\mathbf{x}) \geqslant 9(n+1) \delta^{-1} \log (n+1)+\max \left(h\left(L_{i}^{(v)}\right): v \in S, 0 \leqslant i \leqslant n\right)
\end{gathered}
$$

is contained in the union of not more than

$$
(3 n+3)^{(2 n+2) s} 8^{(n+10)^{2}} \delta^{-(n+1) s-n-5} \log (4 C) \log \log (4 C)
$$

proper linear subspaces of $\mathbb{P}^{n}(\overline{\mathbb{Q}})$ which are all defined over $K$.

Typically, the lower bound for $h(\mathbf{x})$ depends on the linear forms $L_{i}^{(v)}$, while the upper bound for the number of subspaces does not depend on the $L_{i}^{(v)}$.

1.6. We now state an analogue for inequalities with higher degree polynomials instead of linear forms. We first list some notation:

$\delta$ is a real with $0<\delta \leqslant 1, K$ is a number field, $S$ is a finite set of places of $K$ of cardinality $s, X$ is a projective subvariety of $\mathbb{P}^{N}$ defined over $K$ of dimension $n \geqslant 1$ and degree $d, f_{0}^{(v)}, \ldots, f_{n}^{(v)}(v \in S)$ are systems of homogeneous 
polynomials in $\overline{\mathbb{Q}}\left[x_{0}, \ldots, x_{N}\right]$,

$$
\begin{aligned}
& \left\{\begin{array}{l}
C:=\max \left(\left[K\left(f_{i}^{(v)}\right): K\right]: v \in S, i=0, \ldots, n\right), \\
\Delta:=\operatorname{lcm}\left(\operatorname{deg} f_{i}^{(v)}: v \in S, i=0, \ldots, n\right)
\end{array}\right. \\
& \left\{\begin{array}{l}
A_{1}:=\left(20 n \delta^{-1}\right)^{(n+1) s} \cdot \exp \left(2^{12 n+16} n^{4 n} \delta^{-2 n} d^{2 n+2} \Delta^{n(2 n+2)}\right) \\
A_{2}:=(8 n+6)(n+2)^{2} d \Delta^{n+1} \delta^{-1}, \\
A_{3}:=\log (4 C) \log \log (4 C), \\
H:=\log (2 N)+h(X)+\max \left(h\left(1, f_{i}^{(v)}\right): v \in S, 0 \leqslant i \leqslant n\right)
\end{array}\right.
\end{aligned}
$$

Theorem 1.3. Assume that

$$
X(\overline{\mathbb{Q}}) \cap\left\{f_{0}^{(v)}=0, \ldots, f_{n}^{(v)}=0\right\}=\emptyset \quad \text { for } v \in S .
$$

Then there are homogeneous polynomials $G_{1}, \ldots, G_{u} \in K\left[x_{0}, \ldots, x_{N}\right]$ with

$$
u \leqslant A_{1}, \quad \operatorname{deg} G_{i} \leqslant A_{2} \text { for } i=1, \ldots, u
$$

which do not vanish identically on $X$, such that the set of $\mathbf{x} \in X(\overline{\mathbb{Q}})$ with

$$
\log \left(\prod_{v \in S} \prod_{i=0}^{n} \max _{\sigma \in G_{K}} \frac{\left|f_{i}^{(v)}(\sigma(\mathbf{x}))\right|_{v}^{1 / \operatorname{deg} f_{i}^{(v)}}}{\|\sigma(\mathbf{x})\|_{v}}\right) \leqslant-(n+1+\delta) h(\mathbf{x})
$$

is contained in $\bigcup_{i=1}^{u}\left(X \cap\left\{G_{i}=0\right\}\right)$.

Clearly, the bounds in Theorem 1.3 are much worse than those in the result of Evertse and Schlickewei. It would be very interesting if one could replace $A_{1}, A_{3}$ by quantities which are at most exponential in (some power of) $n$ and which are polynomial in $\delta^{-1}, d, \Delta$. Further, we do not know whether the dependence of $A_{2}$ on $\delta$ is needed.

1.7. Our starting point is a result for twisted heights on $\mathbb{P}^{n}$ (a quantitative version of the Absolute Parametric Subspace Theorem), due to Evertse and Schlickewei [5, Theorem 2.1] (see also Proposition 3.1] in Section 3] below). 
From this, we deduce an analogous result for twisted heights on arbitrary projective varieties; the statement of this result is in Section 2 (Theorem 2.1) and its proof in Section 3. The proof involves some arguments from Evertse and Ferretti [4], in particular an explicit lower bound of the normalized Chow weight of a projective variety in terms of the $m$-th normalized Hilbert weight of that variety. In Section 4 we give some height estimates; here we use heavily Rémond's exposé [12. Then in Section 5 we deduce Theorem 1.3. Using that $\mathbb{P}^{N}(K)$ has only finitely many points with height below any given bound, Theorem 1.1 follows at once from Theorem 1.3.

\section{TWisted Heights}

2.1. The quantitative version of the Absolute Parametric Subspace Theorem of Evertse and Schlickewei mentioned in the previous section deals with a class of twisted heights defined on $\mathbb{P}^{n}(\overline{\mathbb{Q}})$ parametrized by a real $Q \geqslant 1$. Roughly speaking, this result states that there are a finite number of proper linear subspaces of $\mathbb{P}^{n}$ such that for every sufficiently large $Q$, the set of points in $\mathbb{P}^{n}(\overline{\mathbb{Q}})$ with small $Q$-height is contained in one of these subspaces. Theorem 2.1 stated below is an analogue in which the points are taken from an arbitrary projective variety instead of $\mathbb{P}^{n}$. Loosely speaking, Theorem 1.3 is proved by defining a suitable finite morphism $\varphi$ from $X$ to a projective variety $Y \subset \mathbb{P}^{R}$ and a finite number of classes of twisted heights on $Y$ as above, and applying Theorem 2.1 to each of these classes.

2.2. Let $K$ be a number field. For finite extensions of $K$ we define normalized absolute values similarly as for $K$. Thus, if $L$ is a finite extension of $K, w$ is a place of $L$, and $v$ is the place of $K$ lying below $w$, then

$$
|x|_{w}=|x|_{v}^{d(w \mid v)} \text { for } x \in K, \text { with } d(w \mid v):=\frac{\left[L_{w}: K_{v}\right]}{[L: K]},
$$

where $K_{v}, L_{w}$ denote the completions at $v, w$, respectively.

We denote points on $\mathbb{P}^{R}$ by $\mathbf{y}=\left(y_{0}, \ldots, y_{R}\right)$. For $v \in M_{K}$, let $\mathbf{c}_{v}=$ $\left(c_{0 v}, \ldots, c_{R v}\right)$ be a tuple of reals such that $c_{0 v}=\cdots=c_{R v}=0$ for all but finitely many places $v \in M_{K}$ and put $\mathbf{c}=\left(\mathbf{c}_{v}: v \in M_{K}\right)$. Further, let $Q$ be 
a real $\geqslant 1$. We define a twisted height on $\mathbb{P}^{R}(\overline{\mathbb{Q}})$ as follows. First put

$$
H_{Q, \mathbf{c}}(\mathbf{y}):=\prod_{v \in M_{K}} \max _{0 \leqslant i \leqslant R}\left(\left|y_{i}\right|_{v} Q^{c_{i v}}\right) \quad \text { for } \mathbf{y}=\left(y_{0}, \ldots, y_{R}\right) \in \mathbb{P}^{R}(K)
$$

by the product formula, this is well-defined on $\mathbb{P}^{R}(K)$. For any finite extension $L$ of $K$ we put

$$
c_{i w}:=c_{i v} \cdot d(w \mid v) \quad \text { for } w \in M_{L}
$$

where $M_{L}$ is the set of places of $L$ and $v$ the place of $K$ lying below $w$. Then for $\mathbf{y} \in \mathbb{P}^{R}(\overline{\mathbb{Q}})$, we define

$$
H_{Q, \mathbf{c}}(\mathbf{y}):=\prod_{w \in M_{L}} \max _{0 \leqslant i \leqslant R}\left(\left|y_{i}\right|_{w} Q^{c_{i w}}\right)
$$

where $L$ is any finite extension of $K$ such that $\mathbf{y} \in \mathbb{P}^{R}(L)$. In view of (2.1) this definition does not depend on $L$.

2.3. Let $Y$ be a (by definition irreducible) projective subvariety of $\mathbb{P}^{R}$ of dimension $n$ and degree $D$, defined over $K$. We recall that there is an up to a constant factor unique polynomial $F_{Y}\left(\mathbf{u}^{(0)}, \ldots, \mathbf{u}^{(n)}\right)$ with coefficients in $K$ in blocks of variables $\mathbf{u}^{(0)}=\left(u_{0}^{(0)}, \ldots, u_{R}^{(0)}\right), \ldots, \mathbf{u}^{(n)}=\left(u_{0}^{(n)}, \ldots, u_{R}^{(n)}\right)$, called the Chow form of $Y$, with the following properties:

$F_{Y}$ is irreducible over $\overline{\mathbb{Q}} ; F_{Y}$ is homogeneous in each block $\mathbf{u}^{(h)}(h=$ $0, \ldots, n)$; and $F_{Y}\left(\mathbf{u}^{(0)}, \ldots, \mathbf{u}^{(n)}\right)=0$ if and only if $Y$ and the hyperplanes $\sum_{i=0}^{R} u_{i}^{(h)} y_{i}=0(h=0, \ldots, n)$ have a $\overline{\mathbb{Q}}$-rational point in common.

It is well-known that the degree of $F_{Y}$ in each block $\mathbf{u}^{(h)}$ is $D$.

Let $\mathbf{c}=\left(c_{0}, \ldots, c_{R}\right)$ be a tuple of reals. Introduce an auxiliary variable $t$ and substitute $t^{c_{i}} u_{i}^{(h)}$ for $u_{i}^{(h)}$ in $F_{Y}$ for $h=0, \ldots, n, i=0, \ldots, R$. Thus we obtain an expression

$$
\begin{aligned}
F_{Y}\left(t^{c_{0}} u_{0}^{(0)}\right. & \left., \ldots, t^{c_{R}} u_{R}^{(0)} ; \ldots ; t^{c_{0}} u_{0}^{(n)}, \ldots, t^{c_{R}} u_{R}^{(n)}\right) \\
& =t^{e_{0}} G_{0}\left(\mathbf{u}^{(0)}, \ldots, \mathbf{u}^{(n)}\right)+\cdots+t^{e_{r}} G_{r}\left(\mathbf{u}^{(0)}, \ldots, \mathbf{u}^{(n)}\right)
\end{aligned}
$$


with $G_{0}, \ldots, G_{r} \in K\left[\mathbf{u}^{(0)}, \ldots, \mathbf{u}^{(n)}\right]$ and $e_{0}>e_{1}>\cdots>e_{r}$. We now define the Chow weight of $Y$ with respect to $\mathbf{c}^{1}$ by

$$
e_{Y}(\mathbf{c}):=e_{0} .
$$

2.4. We formulate our main result for twisted heights. Below, $Y$ is a projective subvariety of $\mathbb{P}^{R}$ of dimension $n \geqslant 1$ and degree $D$, defined over $K$, and $\mathbf{c}_{v}=\left(c_{0 v}, \ldots, c_{R v}\right)\left(v \in M_{K}\right)$ are tuples of reals such that

$$
\begin{aligned}
& c_{i v} \geqslant 0 \text { for } v \in M_{K}, i=0, \ldots, R ; \\
& c_{0 v}=\cdots=c_{R v}=0 \text { for all but finitely many } v \in M_{K} ; \\
& \sum_{v \in M_{K}} \max \left(c_{0 v}, \ldots, c_{R v}\right) \leqslant 1 .
\end{aligned}
$$

Put

$$
E_{Y}(\mathbf{c}):=\frac{1}{(n+1) D}\left(\sum_{v \in M_{K}} e_{Y}\left(\mathbf{c}_{v}\right)\right) .
$$

Further, let $0<\delta \leqslant 1$, and put

$$
\left\{\begin{array}{l}
B_{1}:=\exp \left(2^{10 n+4} \delta^{-2 n} D^{2 n+2}\right) \cdot \log (4 R) \log \log (4 R) \\
B_{2}:=(4 n+3) D \delta^{-1} \\
B_{3}:=\exp \left(2^{5 n+4} \delta^{-n-1} D^{n+2} \log (4 R)\right)
\end{array}\right.
$$

Theorem 2.1. There are homogeneous polynomials $F_{1}, \ldots, F_{t} \in$ $K\left[y_{0}, \ldots, y_{R}\right]$ with

$$
t \leqslant B_{1}, \quad \operatorname{deg} F_{i} \leqslant B_{2} \text { for } i=1, \ldots, t,
$$

which do not vanish identically on $Y$, such that for every real number $Q$ with

$$
\log Q \geqslant B_{3} \cdot(h(Y)+1)
$$

there is $F_{i} \in\left\{F_{1}, \ldots, F_{t}\right\}$ with

$$
\left\{\mathbf{y} \in Y(\overline{\mathbb{Q}}): H_{Q, \mathbf{c}}(\mathbf{y}) \leqslant Q^{E_{Y}(\mathbf{c})-\delta}\right\} \subset Y \cap\left\{F_{i}=0\right\} .
$$

\footnotetext{
${ }^{1}$ The Chow weight is closely related to the degree of contact earlier introduced by Mumford 10. Roughly speaking, the degree of contact of $Y$ with respect to $\mathbf{c}$ is defined for integer tuples $\mathbf{c}$ and it is equal to $e_{r}$ instead of $e_{0}$.
} 


\section{Proof of Theorem 2.1}

3.1. We first recall the quantitative version of the Absolute Parametric Subspace Theorem of Evertse and Schlickewei. As before, $K$ is an algebraic number field and $R, n$ are integers with $R \geqslant n \geqslant 1$. We denote the coordinates on $\mathbb{P}^{n}$ by $\left(x_{0}, \ldots, x_{n}\right)$. Given an index set $I=\left\{i_{0}, \ldots, i_{n}\right\}$ with $i_{0}<\cdots<i_{n}$ and linear forms $L_{j}=\sum_{i=0}^{n} a_{i j} x_{i}(j \in I)$ we write $\operatorname{det}\left(L_{j}: j \in I\right):=\operatorname{det}\left(a_{i, i_{j}}\right)_{i, j=0, \ldots, n}$.

Let $L_{0}, \ldots, L_{R}$ be linear forms in $K\left[x_{0}, \ldots, x_{n}\right]$ with $\operatorname{rank}\left\{L_{0}, \ldots, L_{R}\right\}=$ $n+1$. Further, let $I_{v}\left(v \in M_{K}\right)$ be subsets of $\{0, \ldots, R\}$ of cardinality $n+1$ such that

$$
\operatorname{rank}\left\{L_{i}: i \in I_{v}\right\}=n+1 \quad \text { for } v \in M_{K}
$$

Define

$$
\mathcal{H}:=\prod_{v \in M_{K}} \max _{I}\left|\operatorname{det}\left(L_{i}: i \in I\right)\right|_{v}, \quad \mathcal{D}:=\prod_{v \in M_{K}}\left|\operatorname{det}\left(L_{i}: i \in I_{v}\right)\right|_{v}
$$

here the maximum is taken over all subsets $I$ of $\{0, \ldots, R\}$ of cardinality $n+1$. According to [4, Lemma 7.2] we have

$$
\mathcal{D} \geqslant \mathcal{H}^{1-\left(\begin{array}{l}
R+1 \\
n+1
\end{array}\right)} \text {. }
$$

Let $\mathbf{d}_{v}=\left(d_{i v}: i \in I_{v}\right)\left(v \in M_{K}\right)$ be tuples of reals such that

$$
\begin{aligned}
& d_{i v}=0 \text { for } i \in I_{v} \text { and for all but finitely many } v \in M_{K}, \\
& \sum_{v \in M_{K}} \sum_{i \in I_{v}} d_{i v}=0, \\
& \sum_{v \in M_{K}} \max \left(d_{i v}: i \in I_{v}\right) \leqslant 1
\end{aligned}
$$

and write $\mathbf{d}=\left(\mathbf{d}_{v}: v \in M_{K}\right)$.

We define a twisted height on $\mathbb{P}^{n}(\overline{\mathbb{Q}})$ as follows. For any real number $Q \geqslant 1$ we first put

$$
H_{Q, \mathbf{d}}^{*}(\mathbf{x})=\prod_{v \in M_{K}}\left(\max _{i \in I_{v}}\left|L_{i}(\mathbf{x})\right|{ }_{v} Q^{-d_{i v}}\right) \quad \text { for } \mathbf{x} \in \mathbb{P}^{n}(K) .
$$


More generally, if $L$ is any finite extension of $K$, put

$$
d_{i w}:=d(w \mid v) d_{i v}, \quad I_{w}:=I_{v}
$$

where $v$ is the place of $K$ lying below $w$. Then for $\mathbf{x} \in \mathbb{P}^{n}(\overline{\mathbb{Q}})$ we define

$$
H_{Q, \mathbf{d}}^{*}(\mathbf{x})=\prod_{w \in M_{L}}\left(\max _{i \in I_{w}}\left|L_{i}(\mathbf{x})\right|_{v} Q^{-d_{i w}}\right)
$$

where $L$ is any finite extension of $K$ such that $\mathbf{x} \in \mathbb{P}^{n}(L)$. This is independent of the choice of $L$.

Now the result of Evertse and Schlickewei [5, Theorem 2.1] is as follows:

Proposition 3.1. Let $I_{v}\left(v \in M_{K}\right), \mathbf{d}=\left(\mathbf{d}_{v}: v \in M_{K}\right)$, satisfy (3.1), (3.4), respectively, and let $0<\varepsilon \leqslant 1$.

There are proper linear subspaces $T_{1}, \ldots, T_{t}$ of $\mathbb{P}^{n}$, defined over $K$, with

$$
t \leqslant 4^{(n+9)^{2}} \varepsilon^{-n-5} \log (3 R) \log \log (3 R),
$$

such that for every real number $Q$ with

$$
Q \geqslant \max \left(\mathcal{H}^{1 /\left(\begin{array}{c}
R+1 \\
n+1
\end{array}\right)},(n+1)^{2 / \varepsilon}\right)
$$

there is $T_{i} \in\left\{T_{1}, \ldots, T_{t}\right\}$ with

$$
\left\{\mathbf{x} \in \mathbb{P}^{n}(\overline{\mathbb{Q}}): H_{Q, \mathbf{d}}^{*}(\mathbf{x}) \leqslant \mathcal{D}^{1 /(n+1)} Q^{-\varepsilon}\right\} \subset T_{i} .
$$

3.2. We recall some results from [4. As in Section 2] we denote the coordinates on $\mathbb{P}^{R}$ by $\left(y_{0}, \ldots, y_{R}\right)$. Let $Y$ be a projective variety of $\mathbb{P}^{R}$ defined over $K$ of dimension $n$ and degree $D$. Let $I_{Y}$ be the prime ideal of $Y$, i.e. the ideal of polynomials from $\overline{\mathbb{Q}}\left[y_{0}, \ldots, y_{R}\right]$ vanishing identically on $Y$. For $m \in \mathbb{N}$, denote by $\overline{\mathbb{Q}}\left[y_{0}, \ldots, y_{R}\right]_{m}$ the vector space of homogeneous polynomials in $\overline{\mathbb{Q}}\left[y_{0}, \ldots, y_{R}\right]$ of degree $m$, and put $\left(I_{Y}\right)_{m}:=\overline{\mathbb{Q}}\left[y_{0}, \ldots, y_{R}\right]_{m} \cap I_{Y}$. Then the Hilbert function of $Y$ is defined by

$$
H_{Y}(m):=\operatorname{dim}_{\overline{\mathbb{Q}}}\left(\overline{\mathbb{Q}}\left[y_{0}, \ldots, y_{R}\right]_{m} /\left(I_{Y}\right)_{m}\right) .
$$

The scalar product of $\mathbf{a}=\left(a_{0}, \ldots, a_{R}\right), \mathbf{b}=\left(b_{0}, \ldots, b_{R}\right) \in \mathbb{R}^{R+1}$ is given by $\mathbf{a} \cdot \mathbf{b}:=a_{0} b_{0}+\cdots+a_{R} b_{R}$. For $\mathbf{a}=\left(a_{0}, \ldots, a_{R}\right) \in\left(\mathbb{Z}_{\geqslant 0}\right)^{R+1}$, denote by $\mathbf{y}^{\mathbf{a}}$ 
the monomial $y_{0}^{a_{0}} \cdots y_{R}^{a_{R}}$. Then the $m$-th Hilbert weight of $Y$ with respect to a tuple $\mathbf{c}=\left(c_{0}, \ldots, c_{R}\right) \in \mathbb{R}^{R+1}$ is defined by

$$
s_{Y}(m, \mathbf{c}):=\max \left(\sum_{i=1}^{H_{Y}(m)} \mathbf{a}_{i} \cdot \mathbf{c}\right)
$$

where the maximum is taken over all sets of monomials $\left\{\mathbf{y}^{\mathbf{a}_{1}}, \ldots, \mathbf{y}^{\mathbf{a}_{H_{Y}(m)}}\right\}$, whose residue classes modulo $\left(I_{Y}\right)_{m}$ form a basis of $\overline{\mathbb{Q}}\left[y_{0}, \ldots, y_{R}\right]_{m} /\left(I_{Y}\right)_{m}$.

We recall Evertse and Ferretti [4, Theorem 4.1]:

Proposition 3.2. Let $\mathbf{c}=\left(c_{0}, \ldots, c_{R}\right)$ be a tuple of non-negative reals. Let $m>D$ be an integer. Then

$$
\frac{1}{m H_{Y}(m)} \cdot s_{Y}(m, \mathbf{c}) \geqslant \frac{1}{(n+1) D} \cdot e_{Y}(\mathbf{c})-\frac{(2 n+1) D}{m} \cdot \max \left(c_{0}, \ldots, c_{R}\right) .
$$

Let $m$ be a positive integer. Put

$$
n_{m}:=H_{Y}(m)-1, \quad R_{m}:=\left(\begin{array}{c}
R+m \\
m
\end{array}\right)-1,
$$

and let $\mathbf{y}^{\mathbf{a}_{0}}, \ldots, \mathbf{y}^{\mathbf{a}_{R_{m}}}$ be the monomials of degree $m$ in $y_{0}, \ldots, y_{R}$, in some order. Denote by $\varphi_{m}$ the Veronese map of degree $m, \mathbf{y} \mapsto\left(\mathbf{y}^{\mathbf{a}_{0}}, \ldots, \mathbf{y}^{\mathbf{a}_{R_{m}}}\right)$. Lastly, denote by $Y_{m}$ the smallest linear subspace of $\mathbb{P}^{R_{m}}$ containing $\varphi_{m}(Y)$.

Lemma 3.3. (i) $Y_{m}$ is defined over $K$;

(ii) $\operatorname{dim} Y_{m}=n_{m} \leqslant D\left(\begin{array}{c}m+n \\ n\end{array}\right)$;

(iii) $h\left(Y_{m}\right) \leqslant D m\left(\begin{array}{c}m+n \\ n\end{array}\right)\left(D^{-1} h(Y)+(3 n+4) \log (R+1)\right)$.

Proof. (i),(iii) [4, Lemma 8.3]; (ii) Chardin [1, Théorème 1].

3.3. Let $\mathbf{c}_{v} \in \mathbb{R}^{R}\left(v \in M_{K}\right)$ be tuples with (2.6). For a suitable value of $m$, we link the twisted height $H_{Q, \mathbf{c}}$ from Theorem 2.1 to a twisted height on $\mathbb{P}^{n_{m}}$ to which Proposition 3.1 is applicable. Put

$$
m:=\left[(4 n+3) D \delta^{-1}\right] .
$$

Then by Proposition 3.2 and (2.6) we have

$$
\frac{1}{m H_{Y}(m)} \cdot\left(\sum_{v \in M_{K}} s_{Y}\left(m, \mathbf{c}_{v}\right)\right) \geqslant \frac{1}{(n+1) D} \cdot\left(\sum_{v \in M_{K}} e_{Y}\left(\mathbf{c}_{v}\right)\right)-\frac{\delta}{2} .
$$


Denote as before the coordinates on $\mathbb{P}^{R}$ by $\mathbf{y}=\left(y_{0}, \ldots, y_{R}\right)$, those on $\mathbb{P}^{n_{m}}=\mathbb{P}^{H_{Y}(m)-1}$ by $\mathbf{x}=\left(x_{0}, \ldots, x_{n_{m}}\right)$, and those on $\mathbb{P}^{R_{m}}=\mathbb{P}^{\left(\begin{array}{c}R+m \\ m\end{array}\right)-1}$ by $\mathbf{z}=\left(z_{0}, \ldots, z_{R_{m}}\right)$. Since $Y_{m}$ is an $n_{m}$-dimensional linear subspace of $\mathbb{P}^{R_{m}}$ defined over $K$, there are linear forms $L_{0}, \ldots, L_{R_{m}} \in K\left[x_{0}, \ldots, x_{n_{m}}\right]$ such that the map

$$
\psi_{m}: \mathbf{x} \mapsto\left(L_{0}(\mathbf{x}), \ldots, L_{R_{m}}(\mathbf{x})\right)
$$

is a linear isomorphism from $\mathbb{P}^{n_{m}}$ to $Y_{m}$. Thus, $\psi_{m}^{-1} \varphi_{m}$ is an injective map from $Y$ into $\mathbb{P}^{n_{m}}$.

For $v \in M_{K}$ there is a subset $I_{v}$ of $\left\{0, \ldots, R_{m}\right\}$ of cardinality $n_{m}+1=$ $H_{Y}(m)$ such that $\left\{\mathbf{y}^{\mathbf{a}_{i}}: i \in I_{v}\right\}$ is a basis of $\overline{\mathbb{Q}}\left[y_{0}, \ldots, y_{R}\right]_{m} /\left(I_{Y}\right)_{m}$ and

$$
s_{Y}\left(m, \mathbf{c}_{v}\right)=\sum_{i \in I_{v}} \mathbf{a}_{i} \cdot \mathbf{c}_{v} .
$$

Now define the tuples $\mathbf{d}_{v}=\left(d_{i v}, i \in I_{v}\right)\left(v \in M_{K}\right)$ by

$$
\begin{aligned}
d_{i v} & =-\frac{1}{m} \cdot \mathbf{a}_{i} \cdot \mathbf{c}_{v}+\frac{1}{m\left(n_{m}+1\right)}\left(\sum_{j \in I_{v}} \mathbf{a}_{j} \cdot \mathbf{c}_{v}\right) \\
& =-\frac{1}{m} \cdot \mathbf{a}_{i} \cdot \mathbf{c}_{v}+\frac{1}{m H_{Y}(m)} \cdot s_{Y}\left(m, \mathbf{c}_{v}\right),
\end{aligned}
$$

and put $\mathbf{d}=\left(\mathbf{d}_{v}: v \in M_{K}\right)$. Similarly to (3.2) we define

$$
\mathcal{H}:=\prod_{v \in M_{K}} \max _{I}\left|\operatorname{det}\left(L_{i}: i \in I\right)\right|_{v}, \quad \mathcal{D}:=\prod_{v \in M_{K}}\left|\operatorname{det}\left(L_{i}: i \in I_{v}\right)\right|_{v},
$$

where the maximum is taken over all subsets $I$ of $\left\{0, \ldots, R_{m}\right\}$ of cardinality $n_{m}+1$. Then by, e.g., [4, page 1300] we have

$$
\log \mathcal{H}=h\left(Y_{m}\right)
$$

We define in a usual manner a twisted height on $\mathbb{P}^{n_{m}}(\overline{\mathbb{Q}})$ by putting

$$
H_{Q, \mathbf{d}}^{*}(\mathbf{x})=\prod_{w \in M_{L}} \max _{i \in I_{w}}\left(\left|L_{i}(\mathbf{x})\right|_{w} Q^{-d_{i w}}\right)
$$

for $\mathbf{x} \in \mathbb{P}^{n_{m}}(\overline{\mathbb{Q}})$, where $L$ is any finite extension of $K$ such that $\mathbf{x} \in \mathbb{P}^{n_{m}}(L)$, $Q \geqslant 1$ is a real number, and $d_{i w}=d(w \mid v) d_{i v}, I_{w}=I_{v}$ with $v$ the place of $K$ below $w$. It follows at once from (2.7) that $d_{i v}=0$ for all but finitely many $v$ and for $i \in I_{v}$. Therefore this height is well-defined. 
Lemma 3.4. Assume that

$$
Q \geqslant \mathcal{D}^{6 / \delta m\left(n_{m}+1\right)}
$$

Let $\mathbf{y} \in Y(\overline{\mathbb{Q}})$ be such that

$$
H_{Q, \mathbf{c}}(\mathbf{y}) \leqslant Q^{E_{Y}(\mathbf{c})-\delta}
$$

where $E_{Y}(\mathbf{c})=\frac{1}{(n+1) D}\left(\sum_{v \in M_{K}} e_{Y}\left(\mathbf{c}_{v}\right)\right)$. Let $\mathbf{x}=\psi_{m}^{-1} \varphi_{m}(\mathbf{y})$. Then

$$
H_{Q^{m}, \mathbf{d}}^{*}(\mathbf{x}) \leqslant \mathcal{D}^{1 /\left(n_{m}+1\right)}\left(Q^{m}\right)^{-\delta / 3} .
$$

Proof. Put $s_{v}:=\frac{1}{m H_{Y}(m)} s_{Y}\left(m, \mathbf{c}_{v}\right), s:=\sum_{v \in M_{K}} s_{v}$. We first show that

$$
H_{Q^{m}, \mathbf{d}}^{*}(\mathbf{x}) \leqslant Q^{-m s}\left(H_{Q, \mathbf{c}}(\mathbf{y})\right)^{m} .
$$

Take a finite extension $L$ of $K$ such that $\mathbf{y} \in Y(L)$. We have $\mathbf{x} \in \mathbb{P}^{n_{m}}(L)$ and $L_{i}(\mathbf{x})=\mathbf{y}^{\mathbf{a}_{i}}$ for $i=0, \ldots, R_{m}$. So for $w \in M_{L}$ we have (putting $s_{w}:=d(w \mid v) s_{v}$, with $v$ the place of $K$ below $\left.w\right)$,

$$
\begin{aligned}
& \max _{i \in I_{w}}\left(\left|L_{i}(\mathbf{x})\right|_{w}\left(Q^{m}\right)^{-d_{i w}}\right)=\max _{i \in I_{w}}\left(\left|\mathbf{y}^{\mathbf{a}_{i}}\right|_{w} Q^{\mathbf{a}_{i} \cdot \mathbf{c}_{w}-m s_{w}}\right) \\
& \quad \leqslant \max _{i=0, \ldots, R_{m}}\left(\left|\mathbf{y}^{\mathbf{a}_{i}}\right|_{w} Q^{\mathbf{a}_{i} \cdot \mathbf{c}_{w}-m s_{w}}\right) \leqslant\left(Q^{-s_{w}} \max _{i=0, \ldots, R}\left(\left|y_{i}\right|_{w} Q^{c_{i w}}\right)\right)^{m} .
\end{aligned}
$$

By taking the product over all $w \in M_{L}$, (3.22) follows.

Now a successive application of (3.19), (3.22), (3.20), (3.15) gives

$$
H_{Q^{m}, \mathbf{d}}^{*}(\mathbf{x}) \leqslant \mathcal{D}^{1 /\left(n_{m}+1\right)} Q^{m \delta / 6} \cdot Q^{-m s} Q^{m E_{Y}(\mathbf{c})-m \delta} \leqslant \mathcal{D}^{1 /\left(n_{m}+1\right)}\left(Q^{m}\right)^{-\delta / 3} .
$$

3.4. To complete the proof of Theorem 2.1 we apply Proposition 3.1 to (3.21); that is, we apply Proposition 3.1 with $n=n_{m}, R=R_{m}, \varepsilon=\delta / 3$, and with $Q^{m}$ in place of $Q$. For the moment we assume

$$
\log Q \geqslant \frac{6}{\left(n_{m}+1\right) m \delta}\left(R_{m}+1\right)^{n_{m}+1}\left(h\left(Y_{m}\right)+1\right) .
$$

Notice that this is precisely (3.10) with $R=R_{m}, n=n_{m}, \varepsilon=\delta / 3$ and with $Q^{m}$ in place of $Q$.

We have to verify that (3.1), (3.4), (3.5), (3.6) are satisfied with $n_{m}, R_{m}$ in place of $n, R$. First, (3.1) follows at once from the definition of $I_{v}$ and the fact that $\psi_{m}$ is a linear isomorphism. Second, (3.4) follows from (2.7). 
Third, (3.5) follows from (3.17), (3.16). Last, (3.6) is consequence of (2.6), (2.8) and the fact that $\frac{1}{m H_{Y}(m)} \cdot s_{Y}\left(m, \mathbf{c}_{v}\right)$ can be expressed as a maximum of linear forms in $c_{0 v}, \ldots, c_{R v}$, whose coefficients are non-negative and have sum equal to 1 .

Thus, there are proper linear subspaces $T_{1}, \ldots, T_{t}$ of $\mathbb{P}^{n_{m}}$, defined over $K$, with

$$
t \leqslant 4^{\left(n_{m}+9\right)^{2}}(3 / \delta)^{n_{m}+5} \log \left(3 R_{m}\right) \log \log \left(3 R_{m}\right)
$$

such that for every $Q$ with (3.23) there is $T_{i} \in\left\{T_{1}, \ldots, T_{t}\right\}$ with

$$
\left\{\mathbf{x} \in \mathbb{P}^{n_{m}}(\overline{\mathbb{Q}}): H_{Q^{m}, \mathbf{d}}^{*}(\mathbf{x}) \leqslant \mathcal{D}^{1 /\left(n_{m}+1\right)}\left(Q^{m}\right)^{-\delta / 3}\right\} \subset T_{i} .
$$

For each space $T_{i}$ there is a linear form $L_{i} \in K\left[z_{0}, \ldots, z_{R_{m}}\right]$ vanishing identically on $\psi_{m}\left(T_{i}\right)$ but not on $Y_{m}$. Since by definition, $Y_{m}$ is the smallest linear subvariety of $\mathbb{P}^{R_{m}}$ containing $\varphi_{m}(Y)$, the linear form $L_{i}$ does not vanish identically on $\varphi_{m}(Y)$. Replacing in $L_{i}$ the coordinate $z_{j}$ by $\mathbf{y}^{\mathbf{a}_{j}}$ for $j=0, \ldots, R_{m}$, we obtain a homogeneous polynomial $F_{i} \in K\left[y_{0}, \ldots, y_{R}\right]$ of degree $m$ not vanishing identically on $Y$ such that if $\mathbf{x}=\psi_{m}^{-1} \varphi_{m}(\mathbf{y}) \in T_{i}$, then $F_{i}(\mathbf{y})=0$.

It is easily seen that assumption (3.23), together with (3.18) and (3.3), implies (3.19); hence Lemma 3.4 is applicable. Thus, we infer that there are homogeneous polynomials $F_{1}, \ldots, F_{t} \in K\left[y_{0}, \ldots, y_{R}\right]$ of degree $m$, with $t$ satisfying (3.24), such that for every $Q$ with (3.23) there is $F_{i} \in\left\{F_{1}, \ldots, F_{t}\right\}$ with

$$
\left\{\mathbf{y} \in Y(\overline{\mathbb{Q}}): H_{Q, \mathbf{c}}(\mathbf{y}) \leqslant Q^{E_{Y}(\mathbf{c})-\delta}\right\} \subset Y \cap\left\{F_{i}=0\right\} .
$$

By (3.14) we have $m \leqslant(4 n+3) D \delta^{-1}$, which is the quantity $B_{2}$ from (2.10). So to complete the proof of Theorem 2.1, it suffices to show that the righthand side of (3.24) is at most $B_{1}$ and that the right-hand side of (3.23) is at most $B_{3} \cdot(h(Y)+1)$, where $B_{1}, B_{3}$ are given by (2.10).

Using $m \geqslant 7$ and the inequality

$$
\left(\begin{array}{c}
x+y \\
y
\end{array}\right) \leqslant \frac{(x+y)^{x+y}}{x^{x} y^{y}}=\left(1+\frac{y}{x}\right)^{x} \cdot\left(1+\frac{x}{y}\right)^{y} \leqslant\left(e\left(1+\frac{x}{y}\right)\right)^{y}
$$

for positive integers $x, y$, we infer

$$
R_{m}=\left(\begin{array}{c}
R+m \\
m
\end{array}\right)-1 \leqslant\left(e\left(1+\frac{R}{m}\right)\right)^{m} \leqslant(4 R)^{m}
$$


So by (3.14),

$$
\begin{aligned}
\log \left(3 R_{m}\right) \log \log \left(3 R_{m}\right) & \leqslant 2 m^{2} \log (4 R) \log \log (4 R) \\
& \leqslant 2(8 n+6)^{2} D^{2} \delta^{-2} \log (4 R) \log \log (4 R) .
\end{aligned}
$$

Further, by Lemma 3.3. (ii),

$$
\begin{aligned}
n_{m} & \leqslant D\left(\begin{array}{c}
m+n \\
n
\end{array}\right) \leqslant D\left(e\left(1+\frac{m}{n}\right)\right)^{n} \\
& \leqslant D\left(e\left(1+7 D \delta^{-1}\right)\right)^{n} \leqslant 2^{5 n} \delta^{-n} D^{n+1} .
\end{aligned}
$$

Hence the right-hand side of (3.24) is at most

$$
\begin{gathered}
4^{\left(2^{5 n} \delta^{-n} D^{n+1}+9\right)^{2}}\left(3 \delta^{-1}\right)^{2^{5 n} \delta^{-n} D^{n+1}+5} \\
\cdot 2(8 n+6)^{2} D^{2} \delta^{-2} \log (4 R) \log \log (4 R) \\
\leqslant \exp \left(2^{10 n+4} \delta^{-2 n} D^{2 n+2}\right) \cdot \log (4 R) \log \log (4 R)=B_{1},
\end{gathered}
$$

while by Lemma 3.3, (3.14), (3.26), (3.27), the right-hand side of (3.23) is at most

$$
\begin{aligned}
& \frac{6}{\left(n_{m}+1\right) m \delta}\left((4 R)^{m}+1\right)^{n_{m}+1} \cdot \\
& \cdot\left(1+D m\left(\begin{array}{c}
m+n \\
n
\end{array}\right)\left(D^{-1} h(Y)+(3 n+4) \log (R+1)\right)\right. \\
& \leqslant \quad \delta^{-1}\left((4 R)^{\left.(4 n+3) D \delta^{-1}+1\right)^{2^{5 n} \delta^{-n} D^{n+1}+1} .}\right. \\
& \cdot 2^{5 n} \delta^{-n} D^{n+1}(3 n+1) \log (R+1) \cdot(h(Y)+1) \\
&<\exp \left(2^{5 n+4} \delta^{-n-1} D^{n+2} \log (4 R)\right) \cdot(h(Y)+1)=B_{3} \cdot(h(Y)+1) .
\end{aligned}
$$

This completes the proof of Theorem 2.1 .

\section{Height estimates}

4.1. In this section we compute some height estimates, using Rémond's paper $[12$.

Let $K$ be a number field. Denote as before the set of places of $K$ by $M_{K}$, and denote the sets of archimedean and non-archimedean places of $K$ by $M_{K}^{\infty}$ and $M_{K}^{0}$, respectively. We use the normalized absolute values $|\cdot|_{v}$ introduced in $\$ \mathbf{1 . 4}$. Recall that for each of these absolute values we 
have chosen an extension to $\overline{\mathbb{Q}}$. In particular, for each $v \in M_{K}^{\infty}$ there is an isomorphic embedding $\sigma_{v}: \overline{\mathbb{Q}} \hookrightarrow \mathbb{C}$ such that $|x|_{v}=\left|\sigma_{v}(x)\right|^{\left[K_{v}: \mathbb{R}\right] /[K: \mathbb{Q}]}$ for $x \in \overline{\mathbb{Q}}$.

We represent polynomials as $f=\sum_{\mathbf{m} \in M_{f}} c_{f}(\mathbf{m}) \mathbf{m}$, where the symbol $\mathbf{m}$ denotes a monomial, $M_{f}$ is a finite set of monomials, and $c_{f}(\mathbf{m})\left(\mathbf{m} \in M_{f}\right)$ are the coefficients. For any map $\sigma$ on the field of definition of $f$ we put $\sigma(f):=\sum_{\mathbf{m} \in M_{f}} \sigma\left(c_{f}(\mathbf{m})\right) \mathbf{m}$.

We define norms for polynomials $f_{i}=\sum_{\mathbf{m} \in M_{f_{i}}} c_{f_{i}}(\mathbf{m}) \mathbf{m}(i=1, \ldots, r)$ with complex coefficients:

$$
\begin{aligned}
& \left\|f_{1}, \ldots, f_{r}\right\|:=\max \left(\left|c_{f_{i}}(\mathbf{m})\right|: 1 \leqslant i \leqslant r, \mathbf{m} \in M_{f_{i}}\right) \\
& \left\|f_{1}, \ldots, f_{r}\right\|_{1}:=\sum_{i=1}^{r} \sum_{\mathbf{m} \in M_{f_{i}}}\left|c_{f_{i}}(\mathbf{m})\right|
\end{aligned}
$$

and for polynomials $f_{1}, \ldots, f_{r}$ with coefficients in $\overline{\mathbb{Q}}$ :

$$
\begin{aligned}
& \left\|f_{1}, \ldots, f_{r}\right\|_{v}:=\max \left(\left|c_{f_{i}}(\mathbf{m})\right|_{v}: 1 \leqslant i \leqslant r, \mathbf{m} \in M_{f_{i}}\right) \quad\left(v \in M_{K}\right), \\
& \left\|f_{1}, \ldots, f_{r}\right\|_{v, 1}:=\left\|\sigma_{v}\left(f_{1}\right), \ldots, \sigma_{v}\left(f_{r}\right)\right\|_{1}^{\left[K_{v}: \mathbb{R}\right] /[K: \mathbb{Q}]}\left(v \in M_{K}^{\infty}\right) \\
& \left\|f_{1}, \ldots, f_{r}\right\|_{v, 1}:=\left\|f_{1}, \ldots, f_{r}\right\|_{v}\left(v \in M_{K}^{0}\right) .
\end{aligned}
$$

Lastly, for polynomials $f_{1}, \ldots, f_{r}$ with coefficients in $K$ we define heights

$$
\begin{aligned}
& h\left(f_{1}, \ldots, f_{r}\right):=\log \left(\prod_{v \in M_{K}}\left\|f_{1}, \ldots, f_{r}\right\|_{v}\right), \\
& h_{1}\left(f_{1}, \ldots, f_{r}\right):=\log \left(\prod_{v \in M_{K}}\left\|f_{1}, \ldots, f_{r}\right\|_{v, 1}\right) .
\end{aligned}
$$

More generally, for polynomials $f_{1}, \ldots, f_{r}$ with coefficients in $\overline{\mathbb{Q}}$ we define $h\left(f_{1}, \ldots, f_{r}\right), h_{1}\left(f_{1}, \ldots, f_{r}\right)$ by choosing a number field $K$ containing the coefficients of $f_{1}, \ldots, f_{r}$ and using the above definitions; this is independent of the choice of $K$.

We state without proof some easy inequalities. First, for $\mathbf{x} \in \overline{\mathbb{Q}}^{n+1}$ and $f \in \overline{\mathbb{Q}}\left[x_{0}, \ldots, x_{n}\right]$ homogeneous of degree $D$ we have

$$
\|f(\mathbf{x})\|_{v} \leqslant\|f\|_{v, 1}\|\mathbf{x}\|_{v}^{D} \quad \text { for } v \in M_{K} .
$$


Second, for $\mathbf{x} \in \mathbb{P}^{N}(\overline{\mathbb{Q}})$ and $f_{0}, \ldots, f_{r} \in \overline{\mathbb{Q}}\left[x_{0}, \ldots, x_{N}\right]$ homogeneous of degree $D$ we have

$$
h(\mathbf{y}) \leqslant D h(\mathbf{x})+h_{1}\left(f_{0}, \ldots, f_{r}\right)
$$

where $\mathbf{y}=\left(f_{0}(\mathbf{x}), \ldots, f_{r}(\mathbf{x})\right)$.

Third, if $f \in \overline{\mathbb{Q}}\left[x_{0}, \ldots, x_{n}\right]$ is homogeneous of degree $D$, and if $g_{0}, \ldots, g_{n} \in$ $\overline{\mathbb{Q}}\left[x_{0}, \ldots, x_{m}\right]$ are homogeneous of equal degree, then for the polynomial $f\left(g_{0}, \ldots, g_{n}\right)$, obtained by substituting the polynomial $g_{i}\left(x_{0}, \ldots, x_{m}\right)$ for $x_{i}$ in $f$ for $i=0, \ldots, n$, we have

$$
h_{1}\left(f\left(g_{0}, \ldots, g_{n}\right)\right) \leqslant h_{1}(f)+D h_{1}\left(g_{0}, \ldots, g_{n}\right) .
$$

Last, for $f_{1}, \ldots, f_{r} \in \overline{\mathbb{Q}}\left[x_{1}, \ldots, x_{n}\right]$ we have

$$
h\left(f_{1}, \ldots, f_{r}\right) \leqslant h_{1}\left(f_{1}, \ldots, f_{r}\right) \leqslant h\left(f_{1}, \ldots, f_{r}\right)+\log M,
$$

where $M$ is the number of non-zero coefficients in $f_{1}, \ldots, f_{r}$.

4.2. We define another height for multihomogeneous polynomials. Given a field $\Omega$ and tuples of non-negative integers $\mathbf{l}=\left(l_{0}, \ldots, l_{m}\right)$, we write $\Omega[\mathbf{l}]$ for the set of polynomials with coefficients in $\Omega$ in blocks of variables $\mathbf{z}^{(0)}=$ $\left(z_{0}^{(0)}, \ldots, z_{l_{0}}^{(0)}\right), \ldots, \mathbf{z}^{(m)}=\left(z_{0}^{(m)}, \ldots, z_{l_{m}}^{(m)}\right)$ which are homogeneous in block $\mathbf{z}^{(h)}$ for $h=0, \ldots, m$. For $f \in \Omega[\mathbf{l}]$ we denote by $\operatorname{deg}_{h} f$ the degree of $f$ in block $\mathbf{z}^{(h)}$.

Let

$$
\begin{aligned}
& S(l+1):=\left\{\left(z_{0}, \ldots, z_{l}\right) \in \mathbb{C}^{l+1}:\left|z_{0}\right|^{2}+\cdots+\left|z_{l}\right|^{2}=1\right\}, \\
& S(\mathbf{l}):=S\left(l_{0}+1\right) \times \cdots \times S\left(l_{m}+1\right) .
\end{aligned}
$$

Denote by $\mu_{l+1}$ the unique $U(l+1, \mathbb{C})$-invariant measure on $S(l+1)$ normalized such that $\mu_{l+1}(S(l+1))=1$, and let $\mu_{\mathbf{l}}=\mu_{l_{0}+1} \times \cdots \times \mu_{l_{m}+1}$ be the product measure on $S(\mathbf{l})$. Then for $f \in \mathbb{C}[\mathbf{l}]$ we set

$$
m(f):=\int_{S(\mathbf{l})} \log \left|f\left(\mathbf{z}^{(0)}, \ldots, \mathbf{z}^{(m)}\right)\right| \cdot \mu_{\mathbf{l}}+\frac{1}{2} \sum_{h=0}^{m} \operatorname{deg}_{h} f\left(\sum_{j=1}^{l_{h}} \frac{1}{2 j}\right) .
$$

Given a number field $K$, we define for $f \in K[\mathbf{l}]$,

$$
h^{*}(f):=\sum_{v \in M_{K}^{\infty}} \frac{\left[K_{v}: \mathbb{R}\right]}{[K: \mathbb{Q}]} m\left(\sigma_{v}(f)\right)+\sum_{v \in M_{K}^{0}} \log \|f\|_{v, 1} .
$$


Again, this does not depend on the choice of the number field $K$ containing the coefficients of $f$, so it defines a height on $\overline{\mathbb{Q}}[\mathbf{l}]$. It is not difficult to verify that

$$
h^{*}\left(f_{1} \cdots f_{r}\right)=\sum_{i=1}^{r} h^{*}\left(f_{i}\right) \text { for } f_{1}, \ldots, f_{r} \in \overline{\mathbb{Q}}[\mathbf{l}] .
$$

Lemma 4.1. Let $\mathbf{l}=\left(l_{0}, \ldots, l_{m}\right)$ be a tuple of non-negative integers, and $f \in \overline{\mathbb{Q}}[\mathbf{l}], f \neq 0$. Then

$$
\left|h^{*}(f)-h_{1}(f)\right| \leqslant \sum_{h=0}^{m}\left(\operatorname{deg}_{h} f\right) \log \left(l_{h}+1\right) .
$$

Proof. Put $A:=\prod_{h=0}^{m}\left(l_{h}+1\right)^{\operatorname{deg}_{h} f}$. According to the definitions of $h^{*}$ and $h_{1}$, it suffices to prove that for $f \in \mathbb{C}[\mathbf{l}]$,

$$
\left|m(f)-\log \|f\|_{1}\right| \leqslant \log A .
$$

Using $\left|f\left(\mathbf{z}^{(0)}, \ldots, \mathbf{z}^{(m)}\right)\right| \leqslant\|f\|_{1}$ for $\left(\mathbf{z}^{(0)}, \ldots, \mathbf{z}^{(m)}\right) \in S(\mathbf{l})$ we obtain at once

$$
m(f) \leqslant \log \|f\|_{1}+\frac{1}{2} \sum_{h=0}^{m} \operatorname{deg}_{h} f\left(\sum_{j=1}^{l_{h}} \frac{1}{2 j}\right) \leqslant \log \|f\|_{1}+\log A .
$$

To prove the inequality in the other direction, write $f=\sum_{\mathbf{m} \in M_{f}} c(\mathbf{m}) \mathbf{m}$, where the sum is over a finite number of monomials $\mathbf{m}=\prod_{h=0}^{m} \prod_{j=0}^{l_{h}}\left(z_{j}^{(h)}\right)^{a_{h j}}$ with $\sum_{j=0}^{l_{h}} a_{h j}=\operatorname{deg}_{h} f$ for $h=0, \ldots, m$. For each such monomial we put

$$
\alpha(\mathbf{m}):=\prod_{h=0}^{m} \frac{\left(\operatorname{deg}_{h} f\right) !}{a_{h 0} ! \cdots a_{h, l_{h}} !} .
$$

Then by an argument on [12, pp. 111,112],

$$
\left(\sum_{\mathbf{m} \in M_{f}} \alpha(\mathbf{m})^{-1}|c(\mathbf{m})|^{2}\right)^{1 / 2} \leqslant A^{1 / 2} \exp (m(f)) .
$$

On combining this with the Cauchy-Schwarz inequality and $\sum_{\mathbf{m}} \alpha(\mathbf{m}) \leqslant A$, we obtain

$$
\begin{aligned}
\|f\|_{1} & =\sum_{\mathbf{m} \in M_{f}}|c(\mathbf{m})| \leqslant\left(\sum_{\mathbf{m} \in M_{f}} \alpha(\mathbf{m})\right)^{1 / 2} \cdot\left(\sum_{\mathbf{m} \in M_{f}} \alpha(\mathbf{m})^{-1}|c(\mathbf{m})|^{2}\right)^{1 / 2} \\
& \leqslant A \exp (m(f)) .
\end{aligned}
$$


This proves $\log \|f\|_{1} \leqslant m(f)+\log A$, hence (4.9).

Lemma 4.2. Let $f_{1}, \ldots, f_{r} \in \overline{\mathbb{Q}}[\mathbf{l}]$ and $f=\prod_{i=1}^{r} f_{i}$. Then

$$
h_{1}(f) \leqslant \sum_{i=1}^{r} h_{1}\left(f_{i}\right) \leqslant h_{1}(f)+2 \sum_{h=0}^{m}\left(\operatorname{deg}_{h} f\right) \log \left(l_{h}+1\right) \text {. }
$$

Proof. The first inequality is straightforward while the second follows from Lemma 4.1 and (4.8).

4.3. In this subsection, $X$ is a projective subvariety of $\mathbb{P}^{N}$ of dimension $n \geqslant 1$ and degree $d$ defined over $\overline{\mathbb{Q}}$.

Let $\Delta$ be a positive integer. Denote by $M_{\Delta}$ the collection of all monomials of degree $\Delta$ in the variables $x_{0}, \ldots, x_{N}$. Let $\mathbf{u}^{(h)}=\left(u_{\mathbf{m}}^{(h)}: \mathbf{m} \in M_{\Delta}\right)$ $(h=0, \ldots, n)$ be blocks of variables. There is an up to a constant factor unique, irreducible polynomial $F_{X, \Delta} \in \overline{\mathbb{Q}}\left[\mathbf{u}^{(0)}, \ldots, \mathbf{u}^{(n)}\right]$, called the $\Delta$-Chow form of $X$, having the following property (see [1] ):

$F_{X, \Delta}\left(\mathbf{u}^{(0)}, \ldots, \mathbf{u}^{(n)}\right)=0$ if and only if there is a $\overline{\mathbb{Q}}$-rational point in the intersection of $X$ and the hypersurfaces $\sum_{\mathbf{m} \in M_{\Delta}} u_{\mathbf{m}}^{(h)} \mathbf{m}=0(h=0, \ldots, n)$.

Notice that $F_{X, 1}$ is none other than the Chow form $F_{X}$ of $X$. The form $F_{X, \Delta}$ corresponds to the Chow form $F_{\varphi_{\Delta}(X)}$ of the image of $X$ under the Veronese embedding of degree $\Delta$. It is known that $F_{X, \Delta}$ is homogeneous of degree $\Delta^{n} d$ in $\mathbf{u}^{(h)}$ for $h=0, \ldots, n$.

For a monomial $\mathbf{m}=x_{0}^{a_{0}} \cdots x_{N}^{a_{N}}$ of degree $\Delta$, put $\beta(\mathbf{m})=\Delta ! / a_{0} ! \cdots a_{N} !$. Then the modified Chow form $G_{X, \Delta}\left(\mathbf{u}^{(0)}, \ldots, \mathbf{u}^{(n)}\right)$ is obtained by substitut$\operatorname{ing} \beta(\mathbf{m})^{1 / 2} u_{\mathbf{m}}^{(h)}$ for the variable $u_{\mathbf{m}}^{(h)}$ in the polynomial $F_{X, \Delta}\left(\mathbf{u}^{(0)}, \ldots, \mathbf{u}^{(n)}\right)$. Notice that $G_{X, 1}=F_{X, 1}=F_{X}$. Further, using the estimates $|\beta(\mathbf{m})| \leqslant \Delta$, $|\beta(\mathbf{m})|_{p} \geqslant|\Delta !|_{p}$ for each prime number $p$, one easily obtains

$$
\begin{aligned}
\left|h_{1}\left(F_{X, \Delta}\right)-h_{1}\left(G_{X, \Delta}\right)\right| & \leqslant \frac{1}{2}(n+1) d \Delta^{n} \log (\Delta !) \\
& \leqslant \frac{1}{2}(n+1) d \Delta^{n+1} \log \Delta .
\end{aligned}
$$

The following is a special case of a fundamental result of Rémond [12, Thm. 2, pp. 99,100]:

Lemma 4.3. $h^{*}\left(G_{X, \Delta}\right)=\Delta^{n+1} h^{*}\left(G_{X, 1}\right)=\Delta^{n+1} h^{*}\left(F_{X}\right)$. 
From this we deduce:

Lemma 4.4. $h_{1}\left(F_{X, \Delta}\right) \leqslant \Delta^{n+1} h\left(F_{X}\right)+5(n+1) d \Delta^{n+1} \log (N+\Delta)$.

Proof. Recall that $F_{X, \Delta}$ and $G_{X, \Delta}$ are homogeneous of degree $\Delta^{n} d$ in each block of variables $\mathbf{u}^{(h)}(h=0, \ldots, n)$ and that each of these blocks has $\left(\begin{array}{c}N+\Delta \\ \Delta\end{array}\right) \leqslant(N+\Delta)^{\Delta}$ variables (that is, the number of coefficients of a homogeneous polynomial of degree $\Delta$ in $N+1$ variables). So by (4.10) and Lemma 4.1.

$$
\begin{aligned}
h_{1}\left(F_{X, \Delta}\right) & \leqslant h_{1}\left(G_{X, \Delta}\right)+\frac{1}{2}(n+1) d \Delta^{n+1} \log \Delta \\
& \leqslant h^{*}\left(G_{X, \Delta}\right)+\frac{1}{2}(n+1) d \Delta^{n+1} \log \Delta+(n+1) d \Delta^{n} \log \left(\begin{array}{c}
N+\Delta \\
\Delta
\end{array}\right) \\
& \leqslant h^{*}\left(G_{X, \Delta}\right)+\frac{3}{2}(n+1) d \Delta^{n+1} \log (N+\Delta) .
\end{aligned}
$$

Then using Lemma 4.2, again Lemma 4.1 and inequality (4.5) we obtain

$$
\begin{aligned}
h_{1}\left(F_{X, \Delta}\right) & \leqslant \Delta^{n+1} h^{*}\left(F_{X}\right)+\frac{3}{2}(n+1) d \Delta^{n+1} \log (N+\Delta) \\
& \leqslant \Delta^{n+1} h_{1}\left(F_{X}\right)+\frac{5}{2}(n+1) d \Delta^{n+1} \log (N+\Delta) \\
& \leqslant \Delta^{n+1} h\left(F_{X}\right)+\frac{5}{2}(n+1) d \Delta^{n+1} \log (N+\Delta)+\Delta^{n+1} \log M,
\end{aligned}
$$

where $M$ is the number of non-zero coefficients of $F_{X}$. Since $F_{X}$ is a polynomial in $n+1$ blocks of $N+1$ variables, and homogeneous of degree $d$ in each block, we have, using (3.25)

$$
\begin{aligned}
M & \leqslant\left(\begin{array}{c}
N+d \\
d
\end{array}\right)^{n+1} \leqslant(e(N+1))^{(n+1) d} \\
& \leqslant \exp \left(\frac{5}{2}(n+1) d \log (N+\Delta)\right) .
\end{aligned}
$$

By inserting this into the last inequality, our lemma follows.

We arrive at the following:

Proposition 4.5. Let $g_{0}, \ldots, g_{R}$ be homogeneous polynomials of degree $\Delta$ in $\overline{\mathbb{Q}}\left[x_{0}, \ldots, x_{N}\right]$ such that

$$
X(\overline{\mathbb{Q}}) \cap\left\{g_{0}=0, \ldots, g_{R}=0\right\}=\emptyset .
$$


Let $Y=\varphi(X)$, where $\varphi$ is the morphism on $X$ given by $\mathbf{x} \mapsto\left(g_{0}(\mathbf{x}), \ldots, g_{R}(\mathbf{x})\right)$.

Then

$$
\begin{aligned}
h(Y) \leqslant & \Delta^{n+1} h(X)+(n+1) d \Delta^{n} h_{1}\left(g_{0}, \ldots, g_{R}\right)+ \\
& +5(n+1) d \Delta^{n+1} \log (N+\Delta)+3(n+1) d \Delta^{n} \log (R+1) .
\end{aligned}
$$

Proof. For $j=0, \ldots, R$ write $y_{j}$ for $g_{j}(\mathbf{x})$ and denote by $\mathbf{g}_{j}$ the vector of coefficients of $g_{j}$, i.e., $g_{j}=\sum_{\mathbf{m} \in M_{\Delta}} c_{g_{j}}(\mathbf{m}) \mathbf{m}$ and $\mathbf{g}_{j}=\left(c_{g_{j}}(\mathbf{m}): \mathbf{m} \in M_{\Delta}\right)$. Introduce blocks of variables $\mathbf{v}^{(h)}=\left(v_{0}^{(h)}, \ldots, v_{R}^{(h)}\right)(h=0, \ldots, n)$ and define the polynomial

$$
G\left(\mathbf{v}^{(0)}, \ldots, \mathbf{v}^{(n)}\right):=F_{X, \Delta}\left(\sum_{j=0}^{R} v_{j}^{(0)} \mathbf{g}_{j}, \ldots, \sum_{j=0}^{R} v_{j}^{(n)} \mathbf{g}_{j}\right) .
$$

Then $G\left(\mathbf{v}^{(0)}, \ldots, \mathbf{v}^{(n)}\right)=0$ if and only if $X$ and the hypersurfaces $\sum_{j=0}^{R} v_{j}^{(h)} g_{j}$ $=0(h=0, \ldots, n)$ have a $\overline{\mathbb{Q}}$-rational point in common, if and only if $Y$ and the hyperplanes $\sum_{j=0}^{R} v_{j}^{(h)} y_{j}=0(h=0, \ldots, n)$ have a $\overline{\mathbb{Q}}$-rational point in common, if and only if $F_{Y}\left(\mathbf{v}^{(0)}, \ldots, \mathbf{v}^{(n)}\right)=0$, where $F_{Y}$ is the Chow form of $Y$. Therefore, $G$ is up to a constant factor equal to a power of $F_{Y}$.

Put $A:=(n+1) d \Delta^{n+1} \log (N+\Delta), B:=(n+1) d \Delta^{n} \log (R+1)$. Notice that $G$ has degree $d \Delta^{n}$ in each block $\mathbf{v}^{(h)}$. Further, by (4.4) we have $h_{1}(G) \leqslant h_{1}\left(F_{X, \Delta}\right)+(n+1) d \Delta^{n} h_{1}\left(g_{0}, \ldots, g_{R}\right)+B$. Together with Lemma4.2. Lemma 4.1, this implies

$$
\begin{aligned}
h(Y) & =h\left(F_{Y}\right) \leqslant h_{1}\left(F_{Y}\right) \leqslant h_{1}(G)+2 B \\
& \leqslant h_{1}\left(F_{X, \Delta}\right)+(n+1) d \Delta^{n} h_{1}\left(g_{0}, \ldots, g_{R}\right)+3 B \\
& \leqslant \Delta^{n+1} h(X)+(n+1) d \Delta^{n} h_{1}\left(g_{0}, \ldots, g_{R}\right)+5 A+3 B,
\end{aligned}
$$

proving our Proposition.

\section{Proof of Theorem 1.3.}

5.1. We start with some auxiliary results. We denote the coordinates of $\mathbb{P}^{R}$ by $\mathbf{y}=\left(y_{0}, \ldots, y_{R}\right)$.

Lemma 5.1. Let $Y$ be a projective subvariety of $\mathbb{P}^{R}$ of dimension $n \geqslant 1$ and degree $D$, defined over $\overline{\mathbb{Q}}$. Let $\mathbf{c}=\left(c_{0}, \ldots, c_{R}\right)$ be a tuple of reals. Let 
$\left\{i_{0}, \ldots, i_{n}\right\}$ be a subset of $\{0, \ldots, R\}$ such that

$$
Y(\overline{\mathbb{Q}}) \cap\left\{y_{i_{0}}=0, \ldots, y_{i_{n}}=0\right\}=\emptyset \text {. }
$$

Then

$$
\frac{1}{(n+1) D} \cdot e_{Y}(\mathbf{c}) \geqslant \frac{1}{n+1} \cdot\left(c_{i_{0}}+\cdots+c_{i_{n}}\right) .
$$

Proof. For a subset $I=\left\{k_{0}, \ldots, k_{n}\right\}$ of $\{0, \ldots, R\}$ with $k_{0}<k_{1}<\cdots<$ $k_{n}$, define the bracket

$$
[I]=[I]\left(\mathbf{u}^{(0)}, \ldots, \mathbf{u}^{(n)}\right):=\operatorname{det}\left(u_{k_{j}}^{(i)}\right)_{i, j=0, \ldots, n},
$$

where again $\mathbf{u}^{(h)}$ denotes the block of variables $\left(u_{0}^{(h)}, \ldots, u_{R}^{(h)}\right)$. Let $I_{1}, \ldots, I_{S}$ with $S=\left(\begin{array}{l}R+1 \\ n+1\end{array}\right)$ be all subsets of $\{0, \ldots, R\}$ of cardinality $n+1$. Then the Chow form $F_{Y}$ of $Y$ can be written as a homogeneous polynomial of degree $D$ in $\left[I_{1}\right], \ldots,\left[I_{S}\right]$ :

$$
F_{Y}=\sum_{\mathbf{a} \in A} C(\mathbf{a})\left[I_{1}\right]^{a_{1}} \cdots\left[I_{S}\right]^{a_{S}},
$$

where $A$ is the set of tuples of non-negative integers $\mathbf{a}=\left(a_{1}, \ldots, a_{S}\right)$ with $a_{1}+\cdots+a_{S}=D$ and where $C(\mathbf{a}) \in \overline{\mathbb{Q}}$ for $\mathbf{a} \in A[9$, p. 41, Theorem IV]. For each bracket $[I]$ we have

$$
[I]\left(t^{c_{0}} u_{0}^{(0)}, \ldots, t^{c_{R}} u_{R}^{(0)} ; \ldots ; t^{c_{0}} u_{0}^{(n)}, \ldots, t^{c_{R}} u_{R}^{(n)}\right)=t^{\sum_{i \in I} c_{i}}[I],
$$

therefore,

$$
\begin{aligned}
F_{Y}\left(t^{c_{0}} u_{0}^{(0)}, \ldots,\right. & \left.t^{c_{R}} u_{R}^{(0)} ; \ldots ; t^{c_{0}} u_{0}^{(n)}, \ldots, t^{c_{R}} u_{R}^{(n)}\right) \\
& =\sum_{\mathbf{a} \in A} C(\mathbf{a}) t^{\sum_{j=1}^{S} a_{j}\left(\sum_{i \in I_{j}} c_{i}\right)}\left[I_{1}\right]^{a_{1}} \cdots\left[I_{S}\right]^{a_{S}}
\end{aligned}
$$

Put $\mathbf{e}_{0}:=(1,0, \ldots, 0), \mathbf{e}_{1}:=(0,1, \ldots, 0), \ldots, \mathbf{e}_{R}:=(0,0, \ldots, 1)$. Write $\left\{i_{0}, \ldots, i_{n}\right\}=: I_{1}$. By (5.1) we have $F_{Y}\left(\mathbf{e}_{i_{0}}, \ldots, \mathbf{e}_{i_{n}}\right) \neq 0$. Further,

$$
\left[I_{1}\right]\left(\mathbf{e}_{i_{0}}, \ldots, \mathbf{e}_{i_{n}}\right)=1, \quad[I]\left(\mathbf{e}_{i_{0}}, \ldots, \mathbf{e}_{i_{n}}\right)=0 \text { for } I \neq I_{1} .
$$

Hence in expression (5.3) there is a term $C \cdot\left[I_{1}\right]^{D}$ with $C \in \overline{\mathbb{Q}}^{*}$, and if we substitute $\mathbf{u}^{(j)}=\mathbf{e}_{i_{j}}(j=0, \ldots, n)$ in (5.4) we obtain $C \cdot t^{D\left(c_{i_{0}}+\cdots+c_{i_{n}}\right)}$. That is, one of the numbers $e_{i}$ in (2.4) is equal to $D\left(c_{i_{0}}+\cdots+c_{i_{n}}\right)$. This implies (5.2) at once. 
In addition, we need the following combinatorial lemma, which is a consequence of [3, Lemma 4].

Lemma 5.2. Let $\theta$ be a real with $0<\theta \leqslant \frac{1}{2}$ and let $q$ be a positive integer. Then there exists a set $\mathcal{W}$ of cardinality at most $(e / \theta)^{q-1}$, consisting of tuples $\left(c_{1}, \ldots, c_{q}\right)$ of non-negative reals with $c_{1}+\cdots+c_{q}=1$, with the following property:

for every set of reals $A_{1}, \ldots, A_{q}$ and $\Lambda$ with $A_{j} \leqslant 0$ for $j=1, \ldots, q$ and $\sum_{j=1}^{q} A_{j} \leqslant-\Lambda$, there exists a tuple $\left(c_{1}, \ldots, c_{q}\right) \in \mathcal{W}$ such that

$$
A_{j} \leqslant-c_{j}(1-\theta) \Lambda \text { for } j=1, \ldots, q \text {. }
$$

5.2. In what follows, $K$ is a number field, $S$ a finite set of places of $K$, and $X, N, n, d, s, C, f_{i}^{(v)}(v \in S, i=0, \ldots, n), C, \Delta, A_{1}, A_{2}, A_{3}, H$ are as in Theorem 1.3. We denote the coordinates on $\mathbb{P}^{N}$ by $\mathbf{x}=\left(x_{0}, \ldots, x_{N}\right)$.

Let $f_{0}, \ldots, f_{R}$ be the distinct polynomials among $\sigma\left(f_{j}^{(v)}\right)(v \in S, j=$ $\left.0, \ldots, n, \sigma \in G_{K}\right)$. Then by (1.4),

$$
R \leqslant C(n+1) s-1 .
$$

Let $K^{\prime}$ be the extension of $K$ generated by the coefficients of $f_{0}, \ldots, f_{R}$. Put $g_{i}:=f_{i}^{\Delta / \operatorname{deg} f_{i}}$ for $i=0, \ldots, R$. Thus, $g_{0}, \ldots, g_{R}$ are homogenenous polynomials in $K^{\prime}\left[x_{0}, \ldots, x_{N}\right]$ of degree $\Delta$. Define

$$
\varphi: \mathbf{x} \mapsto\left(g_{0}(\mathbf{x}), \ldots, g_{R}(\mathbf{x})\right), \quad Y:=\varphi(X) .
$$

By assumption (1.2), $\varphi$ is a finite morphism on $X$, and $Y$ is a projective subvariety of $\mathbb{P}^{R}$ defined over $K^{\prime}$. We have

$$
\operatorname{dim} Y=n, \quad \operatorname{deg} Y=: D \leqslant d \Delta^{n} .
$$

We denote places on $K^{\prime}$ by $v^{\prime}$ and define normalized absolute values $|\cdot|_{v^{\prime}}$ on $K^{\prime}$ similarly to $\S\left[1.4\right.$. Further, for every $v^{\prime} \in M_{K^{\prime}}$ we choose an extension of $|\cdot|_{v^{\prime}}$ to $\overline{\mathbb{Q}}$. Since $K^{\prime} / K$ is a normal extension, for every $v^{\prime} \in M_{K^{\prime}}$ there is $\tau_{v^{\prime}} \in G_{K}$ such that

$$
|x|_{v^{\prime}}=\left|\tau_{v^{\prime}}(x)\right|_{v}^{1 / g(v)} \quad \text { for } x \in \overline{\mathbb{Q}}
$$

where $v \in M_{K}$ is the place below $v^{\prime}$ and $g(v)$ is the number of places of $K^{\prime}$ lying above $v$. For each $v^{\prime} \in M_{K^{\prime}}^{\infty}$ there is an isomorphic embedding 
$\sigma_{v^{\prime}}: K^{\prime} \hookrightarrow \mathbb{C}$ such that $|x|_{v^{\prime}}=\left|\sigma_{v^{\prime}}(x)\right|^{\left[K_{v^{\prime}}: \mathbb{R}\right] /\left[K^{\prime}: \mathbb{Q}\right]}$ for $x \in \overline{\mathbb{Q}}$. We define norms $\|\cdot\|_{v^{\prime}},\|\cdot\|_{v^{\prime}, 1}$ for polynomials similarly as in (4.1), with $K^{\prime}, v^{\prime}, \sigma_{v^{\prime}}$ in place of $K, v, \sigma_{v}$.

5.3. For later purposes we estimate from above $h_{1}\left(1, g_{0}, \ldots, g_{R}\right)$ and $h(Y)$. By a straightforward computation we have for $v^{\prime} \in M_{K^{\prime}}^{\infty}$,

$$
\begin{aligned}
& \left\|1, \sigma_{v^{\prime}}\left(g_{0}\right), \ldots, \sigma_{v^{\prime}}\left(g_{R}\right)\right\|_{1} \\
& \quad=1+\sum_{i=0}^{R}\left\|\sigma_{v^{\prime}}\left(g_{i}\right)\right\|_{1} \leqslant 1+\sum_{i=0}^{R}\left\|\sigma_{v^{\prime}}\left(f_{i}\right)\right\|_{1}^{\Delta / \operatorname{deg} f_{i}} \\
& \quad \leqslant 1+\sum_{i=0}^{R}\left(\left(\begin{array}{c}
\operatorname{deg} f_{i}+N \\
\operatorname{deg} f_{i}
\end{array}\right)\left\|\sigma_{v^{\prime}}\left(f_{i}\right)\right\|\right)^{\Delta / \operatorname{deg} f_{i}} \\
& \leqslant(R+2)(N+\Delta)^{\Delta}\left\|1, \sigma_{v^{\prime}}\left(f_{0}\right), \ldots, \sigma_{v^{\prime}}\left(f_{R}\right)\right\|^{\Delta} \\
& \leqslant(R+2)(N+\Delta)^{\Delta} \prod_{i=0}^{R}\left\|1, \sigma_{v^{\prime}}\left(f_{i}\right)\right\|^{\Delta} .
\end{aligned}
$$

So for $v^{\prime} \in M_{K^{\prime}}^{\infty}$ we have

$$
\left\|1, g_{0}, \ldots, g_{R}\right\|_{v^{\prime}, 1} \leqslant\left((R+2)(N+\Delta)^{\Delta}\right)^{\frac{\left[K_{v^{\prime}}^{\prime}: \mathbb{R}\right]}{\left[K^{\prime}: \mathbb{Q}\right]}} \cdot \prod_{i=0}^{R}\left\|1, f_{i}\right\|_{v^{\prime}}^{\Delta}
$$

In an easier manner one obtains for $v^{\prime} \in M_{K^{\prime}}^{0}$,

$$
\left\|1, g_{0}, \ldots, g_{R}\right\|_{v^{\prime}, 1} \leqslant \prod_{i=0}^{R}\left\|1, f_{i}\right\|_{v^{\prime}}^{\Delta}
$$

So by taking the product over $v^{\prime} \in M_{K^{\prime}}$, substituting (5.5), and using that polynomials with conjugate sets of coefficients have the same height,

$$
\begin{gathered}
h_{1}\left(1, g_{0}, \ldots, g_{R}\right) \leqslant \Delta\left(\sum_{i=0}^{R} h\left(1, f_{i}\right)\right)+\Delta \log \left((R+2)(N+\Delta)^{\Delta}\right) \\
\leqslant \Delta C\left(\sum_{v \in S} \sum_{j=0}^{n} h\left(1, f_{j}^{(v)}\right)\right)+\Delta \log (N+\Delta)+\log (3 C n s),
\end{gathered}
$$


and by inserting this estimate into Proposition 4.5 we infer

$$
\begin{aligned}
h(Y) \leqslant & \Delta^{n+1} h(X)+(n+1) d \Delta^{n+1} C \sum_{v \in S} \sum_{j=0}^{n} h\left(1, f_{j}^{(v)}\right)+ \\
& +6(n+1) d \Delta^{n+1} \log (N+\Delta)+4(n+1) d \Delta^{n} \log (3 C n s) .
\end{aligned}
$$

A straightforward computation gives the more tractable estimates

$$
\begin{aligned}
& h_{1}\left(g_{0}, \ldots, g_{R}\right) \leqslant 6 \Delta^{2} C n s \cdot H, \\
& h(Y) \leqslant 25 n^{2} d \Delta^{n+2} C s \cdot H,
\end{aligned}
$$

where $H$ is defined by (1.5).

5.4. We reduce (1.6) to a finite number of systems of inequalities, and then show that each such system leads to an inequality involving a twisted height.

Let $\mathbf{x} \in X(\overline{\mathbb{Q}})$ be a solution of (1.6). For $v \in S$, let $I_{v}$ be the subset of $\{0, \ldots, R\}$ such that $\left\{f_{j}^{(v)}: j=0, \ldots, n\right\}=\left\{f_{i}: i \in I_{v}\right\}$. Put $G_{v}:=$ $\left\|1, g_{0}, \ldots, g_{R}\right\|_{v, 1}$ for $v \in S$. Then

$$
\sum_{v \in S} \sum_{i \in I_{v}} \log \left(\max _{\sigma \in G_{K}} \frac{\left|g_{i}(\mathbf{x})\right|_{v}}{G_{v}\|\sigma(\mathbf{x})\|_{v}^{\Delta}}\right) \leqslant-(n+1+\delta) \Delta h(\mathbf{x}) .
$$

By (4.2), the terms in the sum are $\leqslant 0$. We apply Lemma 5.2 with $q=$ $(n+1) s$ and $\theta=\frac{\delta}{(2 n+2+2 \delta)}=1-\frac{n+1+\delta / 2}{n+1+\delta}$. We infer that there is a set $\mathcal{W}$ with

$$
\# \mathcal{W} \leqslant\left(\frac{e(2 n+2+2 \delta)}{\delta}\right)^{(n+1) s-1} \leqslant\left(17 n \delta^{-1}\right)^{(n+1) s-1}
$$

consisting of tuples of non-negative reals $\left(c_{i v}: v \in S, i \in I_{v}\right)$ with

$$
\sum_{v \in S} \sum_{i \in I_{v}} c_{i v}=1
$$

such that for every solution $\mathbf{x} \in X(\overline{\mathbb{Q}})$ of (1.6) there is a tuple $\left(c_{i v}: v \in\right.$ $\left.S, i \in I_{v}\right) \in \mathcal{W}$ with

$$
\begin{array}{r}
\log \left(\max _{\sigma \in G_{K}} \frac{\left|g_{i}(\sigma(\mathbf{x}))\right|_{v}}{G_{v} \cdot\|\sigma(\mathbf{x})\|_{v}^{\Delta}}\right) \leqslant-c_{i v}\left(n+1+\frac{\delta}{2}\right) \Delta h(\mathbf{x}) \\
\left(v \in S, i \in I_{v}\right) .
\end{array}
$$

Denote by $S^{\prime}$ the set of places of $K^{\prime}$ lying above the places in $S$. Notice that each element of $G_{K}$ acts as a permutation on $g_{0}, \ldots, g_{R}$. Let $v^{\prime} \in S^{\prime}$. 
Write $v$ for the place of $K$ lying below $v^{\prime}$ and let $\tau_{v^{\prime}} \in G_{K}$ be given by (5.7).

Then we define $I_{v^{\prime}} \subset\{0, \ldots, R\}, c_{i, v^{\prime}}\left(i \in I_{v^{\prime}}\right)$ by

$$
\begin{aligned}
& \left\{g_{i}: i \in I_{v^{\prime}}\right\}=\left\{\tau_{v^{\prime}}^{-1}\left(g_{j}\right): j \in I_{v}\right\} \text { for } v^{\prime} \in S^{\prime}, \\
& c_{i, v^{\prime}}:=c_{j v} / g(v) \text { for } v^{\prime} \in S^{\prime}, i \in I_{v^{\prime}},
\end{aligned}
$$

where $j \in I_{v}$ is the index such that $g_{i}=\tau_{v^{\prime}}^{-1}\left(g_{j}\right)$. Further, we put

$$
G_{v^{\prime}}:=\left\|1, g_{0}, \ldots, g_{R}\right\|_{v^{\prime}, 1} \quad \text { for } v^{\prime} \in M_{K^{\prime}} .
$$

Then in view of (15.7), we can rewrite system (15.12) as

$$
\begin{array}{r}
\log \left(\max _{\sigma \in G_{K}} \frac{\left|g_{i}(\sigma(\mathbf{x}))\right|_{v^{\prime}}}{G_{v^{\prime}} \cdot\|\sigma(\mathbf{x})\|_{v^{\prime}}^{\Delta}}\right) \leqslant-c_{i, v^{\prime}}\left(n+1+\frac{\delta}{2}\right) \Delta h(\mathbf{x}) \\
\left(v^{\prime} \in S^{\prime}, i \in I_{v^{\prime}}\right) .
\end{array}
$$

Invoking (5.10), (5.11) we obtain the following:

Lemma 5.3. There is a set $\mathcal{W}^{\prime}$ of cardinality at most $\left(17 n \delta^{-1}\right)^{(n+1) s-1}$, consisting of tuples of non-negative reals $\left(c_{i, v^{\prime}}: v^{\prime} \in S^{\prime}, i \in I_{v^{\prime}}\right)$ with

$$
\sum_{v \in S^{\prime}} \sum_{i \in I_{v^{\prime}}} c_{i, v^{\prime}}=1
$$

with the property that for every $\mathbf{x} \in X(\overline{\mathbb{Q}})$ with (1.6) there is a tuple in $\mathcal{W}^{\prime}$ such that $\mathbf{x}$ satisfies (5.13).

We consider the solutions of a fixed system (5.13). Put

$$
\begin{aligned}
c_{i, v^{\prime}}=0 & \text { for } v^{\prime} \in S^{\prime}, i \in\{0, \ldots, R\} \backslash I_{v^{\prime}} \\
& \text { and } v^{\prime} \in M_{K^{\prime}} \backslash S^{\prime}, i=0, \ldots, R
\end{aligned}
$$

and put $\mathbf{c}_{v^{\prime}}:=\left(c_{0, v^{\prime}}, \ldots, c_{R, v^{\prime}}\right)$ for $v^{\prime} \in M_{K^{\prime}}, \mathbf{c}:=\left(\mathbf{c}_{v^{\prime}}: v^{\prime} \in M_{K^{\prime}}\right)$. Denote by $\mathbf{y}=\left(y_{0}, \ldots, y_{R}\right)$ the coordinates of $\mathbb{P}^{R}$. We define $H_{Q, \mathbf{c}}(\mathbf{y}), E_{Y}(\mathbf{c})$ similarly as (2.3), (2.9), respectively, but with $K^{\prime}$ in place of $K$.

Lemma 5.4. Let $\mathrm{x} \in X(\overline{\mathbb{Q}})$ be a solution of (5.13) satisfying (1.7) and let $\sigma \in G_{K}$. Put

$$
\mathbf{y}:=\varphi(\sigma(\mathbf{x})), \quad Q:=\exp ((n+1+\delta / 2) \Delta h(\mathbf{x}))
$$


Then

$$
H_{Q, \mathbf{c}}(\mathbf{y}) \leqslant Q^{E_{Y}(\mathbf{c})-\frac{\delta}{2(n+2)^{2}}}
$$

Proof. We first estimate from below $E_{Y}(\mathbf{c})$. Let $v^{\prime} \in S^{\prime}$ and write $I_{v^{\prime}}=$ $\left\{i_{0}, \ldots, i_{n}\right\}$. From assumption (1.2), and from the fact that $X$ is defined over $K$ and that $g_{i_{0}}, \ldots, g_{i_{n}}$ are conjugate over $K$ to powers of $f_{0}^{(v)}, \ldots, f_{n}^{(v)}$ where $v \in S$ is the place below $v^{\prime}$, it follows that $X(\overline{\mathbb{Q}}) \cap\left\{g_{i_{0}}=0, \ldots, g_{i_{n}}=0\right\}=\emptyset$. Since $Y=\varphi(X)$, for $\mathbf{y} \in Y(\overline{\mathbb{Q}})$ there is $\mathbf{x} \in X(\overline{\mathbb{Q}})$ with $y_{i}=g_{i}(\mathbf{x})$ for $i=0, \ldots, R$. Hence

$$
Y(\overline{\mathbb{Q}}) \cap\left\{y_{i_{0}}=0, \ldots, y_{i_{n}}=0\right\}=\emptyset .
$$

Now Lemma 5.1 implies

$$
\frac{1}{(n+1) D} \cdot e_{Y}\left(\mathbf{c}_{v^{\prime}}\right) \geqslant \frac{1}{n+1}\left(c_{i_{0}, v^{\prime}}+\cdots+c_{i_{n}, v^{\prime}}\right)=\frac{1}{n+1} \cdot \sum_{i \in I_{v^{\prime}}} c_{i, v^{\prime}} .
$$

This holds for $v^{\prime} \in S^{\prime}$. For $v^{\prime} \notin S^{\prime}$ we have $e_{Y}\left(\mathbf{c}_{v^{\prime}}\right)=0$ by (5.15). By summing over $v^{\prime} \in S^{\prime}$ and using (5.14), we arrive at

$$
E_{Y}(\mathbf{c}) \geqslant \frac{1}{n+1} \text {. }
$$

Now let $\mathbf{x} \in X(\overline{\mathbb{Q}})$ be a solution of (5.13) with (1.7) and let $\sigma \in G_{K}$. Then $\sigma(\mathbf{x})$ is also a solution of (15.13). In fact, by (5.15), $\sigma(\mathbf{x})$ satisfies (5.13) for $v \in M_{K}, i=0, \ldots, R$. Write $\mathbf{y}=\varphi(\sigma(\mathbf{x}))$ so that $y_{i}=g_{i}(\sigma(\mathbf{x}))$ for $i=0, \ldots, R$. Let $L$ be a finite normal extension of $K^{\prime}$ such that $\sigma(\mathbf{x}) \in$ $X(L)$. Pick $w \in M_{L}$ and let $v^{\prime}$ be the place of $K^{\prime}$ below $w$. Then there is $\tau_{w} \in \operatorname{Gal}\left(\overline{\mathbb{Q}} / K^{\prime}\right)$ such that $|x|_{w}=\left|\tau_{w}(x)\right|_{v^{\prime}}^{d\left(w \mid v^{\prime}\right)}$ for $x \in L$, where $d\left(w \mid v^{\prime}\right)=$ $\left[L_{w}: K_{v^{\prime}}^{\prime}\right] /\left[L: K^{\prime}\right]$. Hence for $i=0, \ldots, R$, with the usual notation $c_{i w}=$ $d\left(w \mid v^{\prime}\right) c_{i, v^{\prime}}$

$$
\begin{aligned}
\left|y_{i}\right|_{w} Q^{c_{i w}} & =\left|g_{i}(\sigma(\mathbf{x}))\right|_{w} Q^{c_{i w}}=\left(\left|g_{i}\left(\tau_{w} \sigma(\mathbf{x})\right)\right|_{v^{\prime}} Q^{c_{i, v^{\prime}}}\right)^{d\left(w \mid v^{\prime}\right)} \\
& \leqslant\left(G_{v^{\prime}}\left\|\tau_{w} \sigma(\mathbf{x})\right\|_{v^{\prime}}^{\Delta}\right)^{d\left(w \mid v^{\prime}\right)}=G_{v^{\prime}}^{d(w \mid v)}\|\sigma(\mathbf{x})\|_{w}^{\Delta} .
\end{aligned}
$$

By taking the product over $w \in M_{L}$ and using $h(\sigma(\mathbf{x}))=h(\mathbf{x})$ we obtain

$$
H_{Q, \mathbf{c}}(\mathbf{y}) \leqslant \exp \left(h_{1}\left(1, g_{0}, \ldots, g_{R}\right)\right) \cdot Q^{\frac{1}{n+1+\delta / 2}} .
$$


Now (5.16) follows by observing that by (15.17), assumption (1.7), and (15.8),

$$
\begin{aligned}
\left(E_{Y}\right. & \left.(\mathbf{c})-\frac{\delta}{2(n+2)^{2}}-\frac{1}{n+1+\delta / 2}\right) \log Q \\
& \geqslant\left(\frac{1}{n+1}-\frac{\delta}{2(n+2)^{2}}-\frac{1}{n+1+\delta / 2}\right) \log Q \\
& =\frac{\delta(4 n+6-\delta(n+1))}{4(n+1)(n+2)^{2}} \cdot \Delta h(\mathbf{x}) \geqslant \frac{\delta \Delta}{2(n+2)^{2}} A_{3} H \\
& \geqslant 6 \Delta^{2} C n s H \geqslant h_{1}\left(1, g_{0}, \ldots, g_{R}\right) .
\end{aligned}
$$

5.5. We finish the proof of Theorem 1.3. We apply Theorem 2.1 with $K^{\prime}$, $\frac{\delta}{2(n+2)^{2}}$ in place of $K, \delta$ and, in view of (5.5) and (5.6), with $D \leqslant d \Delta^{n}$ and $R=C(n+1) s-1$. Notice that by (5.14), (15.15), the conditions (2.6), (2.7), (2.8) (with $K^{\prime}$ in place of $K$ ) are satisfied. Denote by $B_{1}^{\prime}, B_{2}^{\prime}, B_{3}^{\prime}$ the quantities obtained by substituting $\frac{\delta}{2(n+2)^{2}}$ for $\delta, C(n+1) s-1$ for $R$, and $d \Delta^{n}$ for $D$ in the quantities $B_{1}, B_{2}, B_{3}$, respectively, defined by (2.10). Recall that if $\mathbf{x}$ satisfies (1.7) then Lemma 5.4 is applicable. Moreover,

$$
\begin{aligned}
\log Q= & \left(n+1+\frac{\delta}{2}\right) \Delta h(\mathbf{x}) \geqslant A_{3} H \\
= & \exp \left(2^{6 n+20} n^{2 n+3} \delta^{-n-1} d^{n+2} \Delta^{n(n+2)} \log (2 C s)\right) \cdot H \\
\geqslant & \exp \left(2^{5 n+4}\left(2(n+2)^{2} \delta^{-1}\right)^{n+1}\left(d \Delta^{n}\right)^{n+2} \log (4 C(n+1) s)\right) . \\
& \quad \cdot\left(26 n^{2} d \Delta^{n+2} C s\right) \cdot H \\
= & B_{3}^{\prime} \cdot\left(26 n^{2} d \Delta^{n+2} C s\right) \cdot H \geqslant B_{3}^{\prime}(h(Y)+1),
\end{aligned}
$$

where the last inequality follows from (5.9). Hence Theorem 2.1 is applicable.

Now Theorem 2.1 and Lemma 5.4 imply that there are homogeneous polynomials $F_{1}, \ldots, F_{t} \in K^{\prime}\left[y_{0}, \ldots, y_{R}\right]$ not vanishing identically on $Y$, with $t \leqslant B_{1}^{\prime}$ and $\operatorname{deg} F_{i} \leqslant B_{2}^{\prime}$ for $i=1, \ldots, t$, with the property that for every solution $\mathbf{x} \in X(\overline{\mathbb{Q}})$ of (15.13) with (1.7), there is $F_{i} \in\left\{F_{1}, \ldots, F_{t}\right\}$ such that $F_{i}(\varphi(\sigma(\mathbf{x})))=0$ for every $\sigma \in G_{K}$. (In fact, taking $Q=\exp ((n+1+$ $\delta / 2) \Delta h(\mathbf{x}))$ it follows from Theorem 2.1 that there is $F_{i}$ with $F_{i}(\mathbf{y})=0$ for 
every $\mathbf{y} \in Y(\overline{\mathbb{Q}})$ with $H_{Q, \mathbf{c}}(\mathbf{y}) \leqslant Q^{E_{Y}(\mathbf{c})-\delta / 2(n+2)^{2}}$, and then by Lemma 5.4 this holds in particular for all points $\left.\mathbf{y}=\varphi(\sigma(\mathbf{x})), \sigma \in G_{K}.\right)$

This means that $\tilde{F}_{i}(\sigma(\mathbf{x}))=0$ for $\sigma \in G_{K}$, where $\tilde{F}_{i}$ is the polynomial obtained by substituting $g_{j}$ for $y_{j}$ in $F_{i}$ for $j=0, \ldots, R$. Notice that $\tilde{F}_{i} \in K^{\prime}\left[x_{0}, \ldots, x_{N}\right], \operatorname{deg} \tilde{F}_{i} \leqslant B_{2}^{\prime} \Delta$, and that $\tilde{F}_{i}$ does not vanish identically on $X$. Write $\tilde{F}_{i}=\sum_{k=1}^{M} \omega_{k} \tilde{F}_{i k}$ where $\omega_{1}, \ldots, \omega_{M}$ is a $K$-basis of $K^{\prime}$, and the $\tilde{F}_{i k}$ are polynomials with coefficients in $K$. We can choose $G_{i} \in\left\{\tilde{F}_{i k}\right.$ : $k=1, \ldots, M\}$ which does not vanish identically on $X$. Now $\sigma\left(\tilde{F}_{i}\right)(\mathbf{x})=0$ for $\sigma \in G_{K}$. Since the polynomials $\tilde{F}_{i k}$ are linear combinations of the polynomials $\sigma\left(\tilde{F}_{i}\right)\left(\sigma \in G_{K}\right)$ it follows that $\tilde{F}_{i k}(\mathbf{x})=0$ for $k=1, \ldots, M$, so in particular $G_{i}(\mathbf{x})=0$.

It follows that there are homogeneous polynomials $G_{1}, \ldots, G_{t} \in$ $K\left[x_{0}, \ldots, x_{N}\right]$ with $t \leqslant B_{1}^{\prime}$ and $\operatorname{deg} G_{i} \leqslant B_{2}^{\prime} \Delta$ for $i=1, \ldots, t$, not vanishing identically on $X$, such that the set of $\mathbf{x} \in X(\overline{\mathbb{Q}})$ with (5.13) and with (1.7) is contained in $\bigcup_{i=1}^{t}\left(X \cap\left\{G_{i}=0\right\}\right)$.

According to Lemma 5.3 there are at most $T:=\left(17 n \delta^{-1}\right)^{(n+1) s-1}$ different systems (15.13), such that every solution $\mathbf{x} \in X(\overline{\mathbb{Q}})$ of (1.6) satisfies one of these systems. Consequently, there are homogeneous polynomials $G_{1}, \ldots, G_{u} \in K\left[x_{0}, \ldots, x_{N}\right]$ not vanishing identically on $X$, with $u \leqslant B_{1}^{\prime} T$ and with $\operatorname{deg} G_{i} \leqslant B_{2}^{\prime} \Delta$ for $i=1, \ldots, u$, such that the set of $\mathbf{x} \in X(\overline{\mathbb{Q}})$ with (1.6), (1.7) is contained in $\bigcup_{i=1}^{u}\left(X \cap\left\{G_{i}=0\right\}\right)$.

Now the proof of Theorem 1.3 is completed by observing that in view of (2.10),

$$
B_{2}^{\prime} \Delta=(4 n+3)\left(d \Delta^{n}\right)\left(2(n+2)^{2} \delta^{-1}\right) \Delta=(8 n+6)(n+2)^{2} d \Delta^{n+1} \delta^{-1}=A_{2}
$$

and

$$
\begin{aligned}
B_{1}^{\prime} T \leqslant & \exp \left(2^{10 n+4}\left(2(n+2)^{2}\right)^{2 n} \delta^{-2 n}\left(d \Delta^{n}\right)^{2 n+2}\right) \cdot \\
& \cdot \log (4(n+1) C s) \log \log (4(n+1) C s) \cdot\left(17 n \delta^{-1}\right)^{(n+1) s-1} \\
\leqslant & \exp \left(2^{12 n+16} n^{4 n} \delta^{-2 n} d^{2 n+2} \Delta^{n(2 n+2)}\right) \cdot \\
& \quad \cdot\left(20 n \delta^{-1}\right)^{(n+1) s} \cdot \log (4 C) \log \log (4 C) \\
= & A_{1}
\end{aligned}
$$




\section{REFERENCES}

[1] M. Chardin, Une majoration de la fonction de Hilbert et ses conséquences pour l'interpolation algébrique, Bull. Soc. Math. France 117 (1989), 305-318.

[2] P. Corvaja, U. Zannier, On a general Thue's equation, Amer. Journal of Math., to appear.

[3] J.-H. Evertse, On equations in $S$-units and the Thue-Mahler equation, Invent. math. 75 (1984), 561-584.

[4] J.-H. Evertse, R.G. Ferretti, Diophantine Inequalities on Projective Varieties, Int. Math. Res. Notices 2002.25 (2002), 1295-1330.

[5] J.-H. Evertse, H.P. Schlickewei, A quantitative version of the Absolute Subspace Theorem, J. reine angew. Math. 548 (2002), 21-127.

[6] G. Faltings, G. Wüstholz, Diophantine approximations on projective spaces, Invent. math.116 (1994), 109-138.

[7] R.G. Ferretti, Mumford's degree of contact and Diophantine approximations, Compos. Math. 121 (2000), 247-262.

[8] R.G. Ferretti, Diophantine approximations and toric deformations, Duke Math. J. 118 (2003), 493-522.

[9] W.V.D. Hodge, D. Pedoe, Methods of algebraic geometry, vol. II, Cambridge Univ. Press, Cambridge, 1952.

[10] D. Mumford, Stability of projective varieties, Enseign. Math. (2) 23 (1977), 39-110.

[11] G. Rémond, Élimination multihomogène. In: Introduction to Algebraic Independence Theory, Chap. 5. LNM 1752, Springer Verlag 2001.

[12] G. Rémond, Géometrie diophantienne multiprojective, In: Introduction to Algebraic Independence Theory, Chap. 7. LNM 1752, Springer Verlag 2001.

[13] H.P. Schlickewei, The §-adic Thue-Siegel-Roth-Schmidt theorem, Arch. Math. 29 (1977), 267-270.

[14] W.M. Schmidt, Norm form equations, Ann. of Math. 96 (1972), 526-551.

[15] W.M. Schmidt, Simultaneous approximation to algebraic numbers by elements of a number field, Monatsh. Math. 79 (1975), 55-66.

[16] W.M. Schmidt, The subspace theorem in diophantine approximation, Compos. Math. 96 (1989), 121-173.

J.-H. Evertse, Universiteit Leiden, Mathematisch Instituut, Postbus 9512, 2300 RA Leiden, The Netherlands

E-mail address: evertse@math.leidenuniv.nl

R.G. Ferretti, Università della Svizzera Italiana, Via Buffi 23, CH-6900

Lugano, Switzerland

E-mail address: roberto.ferretti@lu.unisi.ch 